\title{
LHC bounds on lepton number violation mediated by doubly and singly-charged scalars
}

\author{
Francisco del Águila and Mikael Chala \\ CAFPE and Dpto. de Fúsica Teórica y del Cosmos, \\ Universidad de Granada, E-18071, Granada, Spain \\ E-mail: faguila@ugr.es, miki@ugr.es
}

AbSTRACT: The only possible doubly-charged scalar decays into two Standard Model particles are into pairs of same-sign charged leptons, $\mathrm{H}^{ \pm \pm} \rightarrow l^{ \pm} l^{ \pm}, l=e, \mu, \tau$, or gauge bosons, $\mathrm{H}^{ \pm \pm} \rightarrow W^{ \pm} W^{ \pm}$; being necessary the observation of both to assert the violation of lepton number. However, present ATLAS and CMS limits on doubly-charged scalar production are obtained under specific assumptions on its branching fractions into dileptons only. Although they can be extended to include decays into dibosons and lepton number violating processes. Moreover, the production rates also depend on the type of electroweak multiplet $\mathrm{H}^{ \pm \pm}$belongs to. We classify the possible alternatives and provide the Feynman rules and codes for generating the corresponding signals for pair and associated doubly-charged scalar production, including the leading contribution from the $s$-channel exchange of electroweak gauge bosons as well as the vector-boson fusion corrections. Then, using the same analysis criteria as the LHC collaborations we estimate the limits on the $\mathrm{H}^{ \pm \pm}$mass as a function of the electroweak multiplet it belongs to, and obtain the bounds on the lepton number violating processes $p p \rightarrow \mathrm{H}^{ \pm \pm} \mathrm{H}^{\mp \mp} \rightarrow \ell^{ \pm} \ell^{ \pm} W^{\mp} W^{\mp}$ and $p p \rightarrow \mathrm{H}^{ \pm \pm} \mathrm{H}^{\mp} \rightarrow \ell^{ \pm} \ell^{ \pm} W^{\mp} Z, \ell=e, \mu$, implied by the ATLAS and CMS doubly-charged scalar searches.

Keywords: Higgs Physics, Beyond Standard Model, Neutrino Physics

ARXIV EPRINT: 1311.1510 


\section{Contents}

1 Introduction 1

2 Which kind of new physics are we looking for? $\quad 5$

3 Gauge scalar interactions $\quad 8$

4 Doubly-charged scalar production $\quad 10$

5 Monte Carlo implementation 12

6 Current analyses $\quad 13$

7 Final state-dependent efficiencies and LNV bounds at the LHC 15

8 Conclusions 24

$\begin{array}{lr}\text { A Contact interactions } & 26\end{array}$

$\begin{array}{lr}\text { B Simulation and analyses } & 26\end{array}$

$\begin{array}{lll}\mathrm{C} \mathrm{CL}_{s} \text { method } & 27\end{array}$

\section{Introduction}

No departure from the Standard Model (SM) predictions has been observed at the LHC, yet; being the properties of the recently discovered Higgs boson apparently SM-like, too [19]. This was also preferred by electroweak (EW) precision data [10, 11] (see for previous fits $[12,13]$ and for an up-date [14-16]), thus making the discovery of new physics (NP) in the first LHC run unlikely. What leaves the well-established neutrino masses as the only clear signal of NP beyond the SM, so far [17], if we obviate the cosmological evidence of dark matter.

Neutrinos are massless in the SM because they have no right-handed (RH) counterparts, $\nu_{R i}$, to form Dirac masses and lepton number (LN) is an accidental symmetry protecting them to acquire Majorana masses [18]. Hence, in order to describe neutrino masses we have to add new degrees of freedom to the SM: either RH neutrinos with the corresponding Yukawa couplings giving Dirac masses to neutrinos after EW symmetry breaking $\left(\right.$ EWSB): $\mathcal{L}_{m_{\nu}}^{Y}=-y_{i j} \overline{L_{L i}} \nu_{R j} \tilde{\phi}+$ h.c. $\rightarrow-y_{i j} v \overline{\nu_{L i}} \nu_{R j}+$ h.c., with $v=\left\langle\phi^{0}\right\rangle \sim 174 \mathrm{GeV}$ the Higgs vacuum expectation value $(\mathrm{VEV}) ;^{1}$ or new (heavy) fields, which in particular

\footnotetext{
${ }^{1} L_{L}=\left(\nu_{L}, l_{L}\right)$ and $\phi=\left(\phi^{+}, \phi^{0}\right)$ are the SM lepton and Higgs doublets, respectively, with $\tilde{\phi}=i \sigma_{2} \phi^{*}$ and $\sigma_{2}$ the second Pauli matrix. We write down column doublets in a row for convenience, when no confusion is expected.
} 
may be also $\nu_{R i}$, with couplings violating LN explicitly or spontaneously and generating Majorana masses for the SM neutrinos at some given order in perturbation theory. In this case, upon integration of the heavy modes, the model is described at low energy by an effective Lagrangian with extra higher-order operators, the one of lowest dimension being the Weinberg operator [19], $\mathcal{O}^{(5)}=\left(\overline{L_{L}^{c}} \tilde{\phi}^{*}\right)\left(\tilde{\phi}^{\dagger} L_{L}\right)$, parametrizing the neutrino Majorana masses: ${ }^{2} \mathcal{L}_{m_{\nu}}^{(5)}=-c_{i j}^{(5)} \mathcal{O}_{i j}^{(5)} / \Lambda+$ h.c. $\rightarrow-\left(c_{i j}^{(5)} v^{2} / \Lambda\right) \overline{\nu_{L i}^{c}} \nu_{L j}+$ h.c., with $\Lambda$ the scale of NP.

In this second case there are no new light degrees of freedom to start with; the simplest realizations being characterized at low energy by the very tiny LN violation (LNV) induced by the neutrino Majorana masses, $\left(m_{\nu}\right)_{i j}=2 c_{i j}^{(5)} v^{2} / \Lambda \sim 0.1 \mathrm{eV}$. Whose measurement is the purpose of the next generation of neutrinoless double $\beta$ decay experiments [20] (for recent reviews see [21-23]). ${ }^{3}$ However, the relevant question in the LHC era is if LNV is at the LHC reach. This is to ask if there are new particles with masses $\Lambda \sim \mathrm{TeV}$ (and then $c_{i j}^{(5)} \sim 10^{-11}$ ) with observable LNV signatures [34]. ${ }^{4}$ There is a wide literature dealing with the simplest realizations of this scenario, which are referred to as see-saw mechanisms of type I, II and III and obtained extending the SM with RH neutrinos [39-43], a scalar triplet [44-49] and vector-like fermion triplets [50, 51], respectively. In order to assert the violation of LN at the LHC it is enough to observe final states with non-zero LN, for the LN of the initial state $(p p)$ vanishes. What in practice means observing events with an excess of leptons, or anti-leptons. Among the three see-saw mechanisms, the see-saw of type II gives the cleanest signal because doubly-charged scalars can decay into pairs of same-sign charged leptons, $\Delta^{ \pm \pm} \rightarrow l^{ \pm} l^{ \pm}$, which accumulate around the doubly-charged scalar mass and allow for a very efficient search $[52,53]$; which is not the case for heavy fermions because they decay into an odd number of light fermions $(\geq 3$, if we exclude decays into Higgs bosons decaying in turn into two photons) [54-59], as required by rotational invariance. As a matter of fact, CMS [60] and ATLAS [61] have already set stringent limits on this process, excluding doubly-charged scalar masses $m_{\Delta^{ \pm \pm}}$ranging from 200 to $400 \mathrm{GeV}$, depending on the assumptions on the branching ratios into same-sign dileptons; although they have not reported on the corresponding limits for LNV. In order to obtain the latter, one must look for events with same-sign charged lepton pairs plus EW gauge bosons, including the production of $p p \rightarrow \mathrm{H}^{ \pm \pm} \mathrm{H}^{\mp \mp} \rightarrow l^{ \pm} l^{ \pm} W^{\mp} W^{\mp}$ and $p p \rightarrow \mathrm{H}^{ \pm \pm} \mathrm{H}^{\mp} \rightarrow l^{ \pm} l^{ \pm} W^{\mp} Z{ }^{5}$, what can be done with the same sampling, four and three isolated leptons plus possibly missing transverse momentum, as we discuss below.

First, however, several general comments are worth to emphasize again:

(i) LNV is minuscule, and hence at the LHC the production of LNV particles must be

\footnotetext{
${ }^{2} L_{L}^{c}=\left(\nu_{L}^{c}, l_{L}^{c}\right)$ is the SM lepton doublet with charge-conjugated fields, $\psi_{L}^{c}=\left(\psi_{L}\right)^{c}=C{\overline{\psi_{L}}}^{T}$; analogously $\psi_{R}^{c}=\left(\psi_{R}\right)^{c}=C{\overline{\psi_{R}}}^{T}$.

${ }^{3}$ Although the leading contribution to this process may come from other (higher-order) operators in more elaborated models [24-33].

${ }^{4}$ If the observed baryon asymmetry of the universe originates from leptogenesis, LNV must be at work at some energy, too [35] (see for recent reviews [36-38]).

${ }^{5}$ In the text generic scalar multiplets are denoted by $\mathrm{H}$ and their doubly-charged component by $\mathrm{H}^{ \pm \pm}$. We use $\Delta$ when only referring to the scalar triplet. While charged leptons are denoted by $l(\ell)$ when tau leptons are (not) included.
} 
very suppressed or their decay very slow. As in the former case these would not be observable, they must transform non-trivially under the SM gauge symmetry and hence be produced with EW strength. ${ }^{6}$ (Obviously, singlets can be produced through mixing with non-singlet states but this mechanism is in general suppressed by the corresponding mixing angles. An example is heavy neutrino production through mixing with SM leptons [54-56], which is suppressed because the corresponding mixing angles are bounded to be small by EW precision data (EWPD) $[62] .{ }^{7}$ )

(ii) Thus, LN must be violated in the decays of the new heavy particles, what requires that they have at least two dominant channels with different LN. (Majorana fermions are charge self-conjugated and hence if they decay into a final state with non-zero LN, they do also decay into the charge-conjugated state with opposite LN.)

(iii) We restrict ourselves to SM extensions with LNV scalars because, as stressed above, scalar signatures allow for a more efficient particle reconstruction. Moreover, although the discovery of the Higgs boson proves the fundamental character of the SM scalar sector at low energy, this remains the less known sector of the model. In summary, the experimental observation of neutrino masses together with the outstanding LHC performance make the search for LNV scalars (eventually contributing to neutrino masses) especially timely. In the following we shall extend the see-saw of type II, which is mediated by an $\mathrm{SU}(2)_{L}$ scalar triplet with hypercharge $Y=1$, $\Delta=\left(\Delta^{++}, \Delta^{+}, \Delta^{0}\right)$, to allow for scalar multiplets $\mathrm{H}$ with arbitrary isospin $T$ and hypercharge $Y$ but with a doubly-charged component $\mathrm{H}^{++}$coupling to a pair of samesign charged leptons. This alone fixes the scalar LN equal to -2 but does not stand for LNV. In order to violate LN the scalars must also decay into SM boson pairs and then to final states with vanishing LN. Indeed, the only other possible two-body decay into SM particles of the doubly-charged scalar is into two $W$ bosons, in the scalar triplet case $\Delta^{ \pm \pm} \rightarrow W^{ \pm} W^{ \pm}$. This decay does require the LN breaking by the (small) non-zero $\Delta^{0}$ VEV. In general, once LN is broken, doubly-charged scalars $\mathrm{H}^{ \pm \pm}$ with non-vanishing LN will also decay into $W$ pairs at some order in perturbation theory; whether its neutral partner $\mathrm{H}^{0}$, if it exists, gets a (small) non-zero VEV, or through mixing with other (heavier) scalar multiplets with diboson couplings. The consideration of doubly-charged scalar decays into dileptons and dibosons on

\footnotetext{
${ }^{6}$ We assume that the new fields do not carry color because we search for LNV particles which mainly manifest as dileptonic resonances.

${ }^{7}$ Even if vector boson fusion contributions are large [63], EWPD including LHC data on the SM Higgs further reduce the limits on lepton mixing [16] and hence, the LHC potential for heavy neutrino detection [54-56]. In any case, LHC direct limits on heavy neutrino production provide independent evidence and restrict the allowed range of heavy neutrino masses [64,65], which are indirectly not accessible to lowest order in the expansion in the small lepton mixing. On the other hand, the LHC reach for heavy neutrino detection can be much larger in the presence of new interactions. In particular, if parity is restored [66-68] and the new charged gauge boson $W^{\prime}$ has a mass of several $\mathrm{TeV}$, heavy Majorana neutrinos and hence LNV events can be observed up to neutrino masses near the $W^{\prime}$ mass [69-71]. In fact, CMS has already set significant bounds on this process [72].
} 
the same footing in order to search for (bound) LNV at the LHC also generalizes previous phenomenological studies.

In simple models the region of parameter space where the doubly-charged scalar branching ratio into two same-sign leptons is comparable to the branching ratio into gauge bosons is small. In general, one of the two couplings is larger than the other and therefore the corresponding decay dominates. However, both decays can naturally have a similar rate in more elaborated models $[24-27,29-32,73,74]{ }^{8}$

(iv) We extend the SM with an extra TeV scalar multiplet at a time, neglecting possible mixing effects with other heavier scalar multiplets except to allow for the decay of the $\mathrm{TeV}$ scalar multiplet into gauge bosons. The only models we shall work out in detail are those with scalar multiplets with components of charge 2 at most, which are those of smaller isospin, too. ${ }^{9}$

$(v)$ We will not discuss flavor constraints either because they are model dependent at a large extent. Thus, although in the see-saw of type II neutrino masses are proportional to the corresponding doubly-charged scalar decays [52, 53, 80,81], in general they are not closely related and more elaborated models can accommodate both independently of their specific values. At any rate, along this paper our approach to LHC searches will be mainly phenomenological and hence largely model independent.

(vi) Once LNV is observed the question will be which its origin is. In the case of doublycharged scalar production under consideration one would like to determine the type of multiplet the doubly-charged scalar belongs to. This can be done sampling appropriately the events with four and three isolated leptons, as has been proposed in [82-84].

In next section we characterize the scalar multiplets with doubly-charged components decaying into pairs of same-sign charged leptons, i.e., the possible isospin and hypercharge multiplet assignments. In general, the larger their isospin is, the higher the dimension of the operators parametrizing the heavy scalar decay and hence smaller their decay rate. Their gauge interactions are detailed in section 3, where we work out the corresponding Feynman rules. Both sections are more technical and can be skipped if the reader is only interested

\footnotetext{
${ }^{8}$ We also assume that the mass splitting between the different components of the multiplet is small and hence the mixing with heavier scalar multiplets and with the SM Higgs. Otherwise, cascade decays within the multiplet (of electroweak strength) would be overwhelming [75-77]. Anyway, if $\mathrm{H}^{ \pm \pm}$mainly decays into $\mathrm{H}^{ \pm} W^{ \pm *}$, their subsequent leptonic decays also involve neutrinos, then making more difficult (less efficient) to reconstruct the doubly-charged scalar [78]. Moreover, the final fermions are softer and do not exhibit the resonant behavior in the same-sign dilepton channel. We will not further consider this scenario in the following.

${ }^{9}$ Multiplets with components with larger charges also have other striking signatures, for instance $\mathrm{H}^{+++} \rightarrow \mathrm{H}^{++*} W^{+*} \rightarrow l^{+} l^{+} l^{+} \nu$, but with less energetic charged leptons in the final state [73, 74, 79]. In any case doubly-charged scalars are in general pair and associated produced with a comparable crosssection, decaying besides into same-sign dileptons and dibosons as assumed here and hence with harder charged leptons in the final state, which make easier (more efficient) the doubly-charged scalar reconstruction.
} 
in the phenomenological implications. The production mechanisms are discussed in section 4. The dominant mechanism for doubly-charged scalar pair and associated production is through the $s$-channel exchange of EW gauge bosons. Vector-boson fusion contributions staying below $10 \%$ for the scalar masses of interest. The software implementation for Monte Carlo simulations is described in section 5, being available upon request. Section 6 contains the analyses mimicking those performed by the ATLAS and CMS experiments; and we extend them to estimate the bounds on LNV in section 7. In particular, we provide a table with an estimate of the efficiencies for the reconstruction of the different decay modes, which allows to derive the corresponding limits on doubly-charged scalar production for any set of branching ratios and hence model. We conclude in section 8. In appendices A, B and $\mathrm{C}$ we gather further technical details on effective operators for doubly-charged scalar production at hadron colliders, the Monte Carlo implementation for doubly-charged scalar pair and associated production and the applied statistics, respectively.

\section{Which kind of new physics are we looking for?}

We want to search for scalar resonances that may decay into a pair of same-sign charged leptons, $\mathrm{H}^{ \pm \pm} \rightarrow l^{ \pm} l^{ \pm}$, and be eventually at the LHC reach. This means to classify the EW multiplets $\mathrm{H}$ which the corresponding doubly-charged scalars can belong to. In general, no matter what $\mathrm{SU}(2)_{L} \times \mathrm{U}(1)_{Y}$ multiplet including $\mathrm{H}^{ \pm \pm}$is considered, one can always write down gauge invariant effective operators giving rise to these decays after EWSB [82]. In fact, this can be done for any of the three lepton bilinears with non-vanishing LN available in the SM: $\overline{L_{L}^{c}} L_{L}, \overline{l_{R}^{c}} l_{R}$ and $\overline{L_{L}^{c}} l_{R}$, the three of them containing the product of two samesign charged leptons $l^{-} l^{-}$. Although we can restrict ourselves to the first two combinations because the operators involving the third one are not independent of those built with the first two: the third combination $\overline{L_{L}^{c}} l_{R}$ requires a $\gamma_{\mu}$ insertion because of the fermions' chirality, and hence the presence of a covariant derivative to ensure the operator is Lorentz invariant; then using integration by parts and the equations of motion, the corresponding operators can be seen to be equivalent to the ones involving $\overline{L_{L}^{c}} L_{L}$ and $\overline{l_{R}^{c}} l_{R}$.

In practice we assume that there is a more fundamental theory reducing at lower energy to the SM plus an extra scalar multiplet $\mathrm{H}$ near the $\mathrm{TeV}$ scale with $\mathrm{LN}=-2$ and a doubly-charged component $\mathrm{H}^{++} .{ }^{10}$ Hence, its isospin $T$ and hypercharge $Y$ must fulfill

$$
T^{\mathrm{H}} \geq\left|T_{3}^{\mathrm{H}^{++}}=2-Y^{\mathrm{H}}\right|
$$

and for any pair of isospin and hypercharge assignments satisfying this relation there is a tower of gauge invariant operators involving $\mathrm{H}$, any of the two bilinears with $\mathrm{LN}=2$, $\overline{L_{L}^{c}} L_{L}$ or $\overline{l_{R}^{c}} l_{R}$, and an increasing number of Higgs doublets $\phi(\tilde{\phi})$, with vanishing LN. This reflects the fact that any $\mathrm{SU}(2)_{L} \times \mathrm{U}(1)_{Y}$ representation satisfying eq. (2.1) can be obtained from the Clebsch-Gordan series of the product of a large enough number of fundamental representations $\phi(\tilde{\phi})$, with $T=1 / 2$ and $Y=1 / 2(-1 / 2)$. In particular, one can correlate

\footnotetext{
${ }^{10}$ Doubly-charged fermions and vector-bosons have been also considered but in other context [85].
} 
the operators involving $\overline{\tilde{L}_{L}} \tau^{a} L_{L},{ }^{11}$ with $T=1$ and $Y=-1$, to those involving $\overline{l_{R}^{c}} l_{R}$, with $T=0$ and $Y=-2$, contracting the former with $\phi^{\dagger} \tau^{-a} \tilde{\phi}$; and vice-versa multiplying by $\tilde{\phi}^{\dagger} \tau^{a} \phi$. However, for any given $\mathrm{H}$ only the operators of lowest dimension in general matter because they are the ones formally giving the largest contributions to the dileptonic $\mathrm{H}^{++}$ decays after EWSB.

For illustration purposes in the following we restrict ourselves to scalar multiplets with at most doubly-charged components:

$$
T^{\mathrm{H}}=T_{3}^{\mathrm{H}^{++}} \leq 2
$$

This stands for an $\mathrm{SU}(2)_{L}$ singlet $\kappa^{++}$with hypercharge $2[29,32,86]$, a doublet $\chi=$ $\left(\chi^{++}, \chi^{+}\right)$with $Y=3 / 2[87-90]$, a triplet $\Delta=\left(\Delta^{++}, \Delta^{+}, \Delta^{0}\right)$ with $Y=1$ mediator of the see-saw of type II [44-49], a quadruplet $\Sigma=\left(\Sigma^{++}, \Sigma^{+}, \Sigma^{0}, \Sigma^{\prime-}\right)$ with $Y=1 / 2$ [91], and a quintuplet which we will assume to be real $\Omega=\left(\Omega^{++}, \Omega^{+}, \Omega^{0}, \Omega^{-}, \Omega^{--}\right)$with $Y=0$. The lowest order gauge invariant operators coupling the doubly-charged component of these multiplets to a pair of same-sign charged leptons after EWSB are of dimension 4 for $\kappa$ and $\Delta, 5$ for $\chi$ and $\Sigma$, and 6 for $\Omega$, respectively [82]:

$$
\begin{aligned}
\mathcal{O}_{\kappa} & =\overline{l_{R}^{c}} l_{R} \kappa ; \quad \mathcal{O}_{\Delta}=\left(\overline{\tilde{L}_{L}} \tau^{a} L_{L}\right) M_{a b}^{\Delta} \Delta^{b}, \quad \text { with } a, b=1,0,-1 ; \\
\mathcal{O}_{\chi}^{(1)} & =\overline{l_{R}^{c}} l_{R}\left(\tilde{\phi}^{\dagger} \chi\right) ; \quad \mathcal{O}_{\chi}^{(2)}=\left(\overline{\tilde{L}_{L}} \tau^{a} L_{L}\right) M_{a b}^{\chi}\left(\phi^{\dagger} \tau^{b} \chi\right), \quad \text { with } a, b=1,0,-1 ; \\
\mathcal{O}_{\Sigma} & =\left(\overline{\tilde{L}_{L}} \tau^{a} L_{L}\right) M_{c, a b}^{\Sigma} \phi^{b} \Sigma^{c}, \quad \text { with } a=1,0,-1, \quad b= \pm \frac{1}{2}, \quad c=\frac{3}{2}, \frac{1}{2},-\frac{1}{2},-\frac{3}{2} \\
\mathcal{O}_{\Omega} & =\left(\overline{\tilde{L}_{L}} \tau^{a} L_{L}\right) M_{c, a b}^{\Omega}\left(\tilde{\phi}^{\dagger} \tau^{b} \phi\right) \Omega^{c}, \quad \text { with } a, b=1,0,-1, \quad c=2,1,0,-1,-2
\end{aligned}
$$

where a sum on repeated indices is understood and we have omitted family indices. $M^{\mathrm{H}}$ are matrices with only non-zero entries for $a+b=0$ if $\mathrm{H}=\Delta$ or $\chi$, and for $a+b+c=0$ when $H=\Sigma$ or $\Omega$ :

$$
\begin{aligned}
M_{a b}^{\Delta} & =\left(\begin{array}{lll}
0 & 0 & 1 \\
0 & 1 & 0 \\
1 & 0 & 0
\end{array}\right) ; & M_{a b}^{\chi} & =\left(\begin{array}{ccc}
0 & 0 & 1 \\
0 & -1 & 0 \\
1 & 0 & 0
\end{array}\right) ; \\
M_{\frac{3}{2}, a b}^{\Sigma} & =\left(\begin{array}{cc}
0 & 0 \\
0 & 0 \\
0 & -1
\end{array}\right), & M_{\frac{1}{2}, a b}^{\Sigma}=\left(\begin{array}{cc}
0 & 0 \\
0 & -\sqrt{\frac{2}{3}} \\
\frac{1}{\sqrt{3}} & 0
\end{array}\right), & M_{-c,-a-b}^{\Sigma}=-M_{c, a b}^{\Sigma} ; \\
M_{2, a b}^{\Omega} & =\left(\begin{array}{ccc}
0 & 0 & 0 \\
0 & 0 & 0 \\
0 & 0 & 1
\end{array}\right), & M_{1, a b}^{\Omega} & =\left(\begin{array}{ccc}
0 & 0 & 0 \\
0 & 0 & \frac{1}{\sqrt{2}} \\
0 & \frac{1}{\sqrt{2}} & 0
\end{array}\right),
\end{aligned}
$$

\footnotetext{
${ }^{11}$ Where $\tilde{L}_{L}=i \sigma_{2} L_{L}^{c}$ and $\tau^{a}$ are the Pauli matrices in the spherical basis, $A^{+1}=-\frac{1}{\sqrt{2}}\left(A_{1}-i A_{2}\right), A^{0}=$ $A_{3}, A^{-1}=\frac{1}{\sqrt{2}}\left(A_{1}+i A_{2}\right)$, times the Clebsch-Gordan coefficients $\mathrm{C}_{a,-a}^{1 \times 1 \rightarrow 0}$, up to a global factor and sign: $\tau^{ \pm 1}= \pm\left(\sigma_{1} \mp i \sigma_{2}\right) / 2, \tau^{0}=\sigma_{3} / \sqrt{2}$.
} 


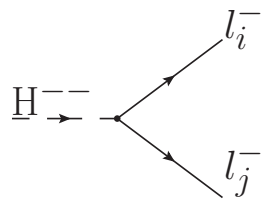

$$
2 i\left[\alpha_{i j}^{L *} P_{L}+\alpha_{i j}^{R *} P_{R}\right]
$$

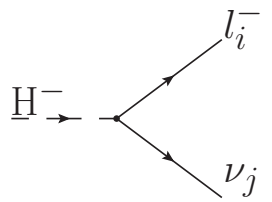

$2 i \beta_{i j}^{*} P_{L}$

Figure 1. Feynman diagrams and rules for Yukawa interactions. The arrows indicate the LN flow.

\begin{tabular}{|lccccc|}
\hline Vertex & Singlet & Doublet & Triplet & Quadruplet & Quintuplet \\
\hline$\alpha_{i j}^{L}$ & 0 & $-\frac{v}{\Lambda} c_{\chi i j}^{(2)}$ & $-c_{\Delta i j}$ & $\frac{v}{\Lambda} c_{\Sigma i j}$ & $\frac{v^{2}}{\Lambda^{2}} c_{\Omega i j}$ \\
\hline$\alpha_{i j}^{R}$ & $c_{\kappa i j}$ & $\frac{v}{\Lambda} c_{\chi i j}^{(1)}$ & 0 & 0 & 0 \\
\hline$\beta_{i j}$ & 0 & $\frac{v}{\Lambda} \frac{c_{\chi i j}^{(2)}}{\sqrt{2}}$ & $\frac{c_{\Delta i j}}{\sqrt{2}}$ & $-\frac{v}{\Lambda} \frac{c_{\Sigma i j}}{\sqrt{3}}$ & $-\frac{v^{2}}{\Lambda^{2}} \frac{c_{\Omega i j}}{2}$ \\
\hline
\end{tabular}

Table 1. Trilinear Scalar-Fermion-Fermion $(\mathcal{S F F})$ couplings for the different multiplet assignments in eq. (2.5).

After EWSB the resulting Yukawa interactions in figure 1 write

$$
\frac{c_{\mathrm{H} i j}}{\Lambda^{n_{\mathrm{H}}}} \mathcal{O}_{\mathrm{H} i j} \rightarrow\left(\alpha_{i j}^{L} \overline{l_{L i}^{c}} l_{L j}+\alpha_{i j}^{R} \overline{l_{R i}^{c}} l_{R j}\right) \mathrm{H}^{++}+\beta_{i j}\left(\overline{\nu_{L i}^{c}} l_{L j}+\overline{l_{L i}^{c}} \nu_{L j}\right) \mathrm{H}^{+}+\cdots
$$

where the couplings $\alpha$ and $\beta$ are in general symmetric, flavor-dependent and suppressed by powers of $v / \Lambda$, as shown in table 1 . Thus, doubly-charged scalars can always couple to same-sign charged lepton pairs in a gauge invariant way independently of the EW multiplet they belong to, although in general with suppressed coefficients. On the other hand, doubly and singly-charged scalar decays are a priori related, even though in practice these relations only have phenomenological implications in quite specific models, as we shall argue later.

There can be also operators of the same order but, for instance, quadratic in the scalar fields. However, they are in general further suppressed. For example, in the quadruplet case the $L L$ interaction in eq. (2.5) can be also obtained from the dimension-5 operator $\mathcal{O}_{\Sigma \otimes \Sigma}=\left(\Sigma^{\dagger} O_{a} \Sigma\right)\left(\tilde{L}_{L} \tau^{a} L_{L}\right)$ (where $O_{a}$ are $4 \times 4$ matrices projecting the $\Sigma \otimes \Sigma$ product into the triplet representation), once the neutral $\Sigma$ component gets a VEV, $\left\langle\Sigma^{0}\right\rangle=v_{\Sigma}$. However, this VEV has to be rather small $\left(v_{\Sigma}<\right.$ few $\left.\mathrm{GeV}\right)$ in order to satisfy, for instance, the constraint on the rho parameter $\left(\rho=1.0004_{-0.0004}^{+0.0003}\right.$ at the $95 \%$ C.L. [17] $) .{ }^{12}$ What in general justifies neglecting the contribution of this operator.

${ }^{12}$ As can be derived from its generic expression (to lowest order in perturbation theory)

$$
\rho=\frac{\sum_{k}\left[T_{k}\left(T_{k}+1\right)-Y_{k}^{2}\right] v_{k}^{2}}{\sum_{k} 2 Y_{k}^{2} v_{k}^{2}}
$$

where $k$ labels the scalar multiplets in the model, and $T_{k}, Y_{k}$ and $v_{k}$ are the corresponding isospin, hypercharge and VEV, respectively. 


\section{Gauge scalar interactions}

Scalar multiplets, $\mathrm{H}$, with doubly-charged components, $\mathrm{H}^{++}$, transform non-trivially under the EW gauge group and thus couple to $\gamma, Z$ and $W$ (except in the singlet case which only has neutral interactions). The explicit form of the gauge couplings is derived from the corresponding kinetic Lagrangian

$$
\mathcal{L}^{K}=\left(D^{\mu} \mathrm{H}\right)^{\dagger} D_{\mu} \mathrm{H}
$$

where the action of the covariant derivative $D_{\mu}$ reads (in standard notation)

$$
\begin{aligned}
D_{\mu} \mathrm{H} & =\left(\partial_{\mu}+i g \vec{T} \cdot \vec{W}_{\mu}+i g^{\prime} Y B_{\mu}\right) \mathrm{H} \\
& =\left(\partial_{\mu}+\frac{i g}{\sqrt{2}}\left(T^{+} W_{\mu}^{+}+T^{-} W_{\mu}^{-}\right)+\frac{i g}{c_{W}}\left(T_{3}-s_{W}^{2} Q\right) Z_{\mu}+i e Q A_{\mu}\right) \mathrm{H}
\end{aligned}
$$

with $s_{W}\left(c_{W}\right)$ the sine (cosine) of the EW mixing angle, $s_{W}=g^{\prime} / \sqrt{g^{2}+g^{\prime 2}}, e=g s_{W}$ the (positive) electromagnetic gauge coupling and $Q=T_{3}+Y$ the electric charge operator. In particular, expanding $\mathcal{L}^{K}$ and reordering terms, the trilinear and quartic gauge couplings involved in the calculation of the pair and associated production of doubly-charged scalars can be written

$$
\begin{aligned}
\mathcal{L}^{K} & \rightarrow\left\{i \frac{g}{\sqrt{2}} \sqrt{(T-Y+2)(T+Y-1)} W_{\mu}^{-}\left[\mathrm{H}^{++}\left(\partial^{\mu} \mathrm{H}^{-}\right)-\left(\partial^{\mu} \mathrm{H}^{++}\right) \mathrm{H}^{-}\right]\right. \\
& +i\left[2 e A_{\mu}+\frac{g}{c_{W}}\left(2-Y-2 s_{W}^{2}\right) Z_{\mu}\right] \mathrm{H}^{++}\left(\partial^{\mu} \mathrm{H}^{--}\right) \\
& \left.+i\left[e A_{\mu}+\frac{g}{c_{W}}\left(1-Y-s_{W}^{2}\right) Z_{\mu}\right] \mathrm{H}^{+}\left(\partial^{\mu} \mathrm{H}^{-}\right)+\text {h.c. }\right\} \\
& +g^{2}\left[T(T+1)-(2-Y)^{2}\right] W_{\mu}^{+} W^{-\mu} \mathrm{H}^{++} \mathrm{H}^{--} \\
& +\left\{\frac{g}{\sqrt{2}} \sqrt{(T-Y+2)(T+Y-1)} W_{\mu}^{-}\left[3 e A^{\mu}+\frac{g}{c_{W}}\left(3-2 Y-3 s_{W}^{2}\right) Z^{\mu}\right] \mathrm{H}^{++} \mathrm{H}^{-}+\text {h.c. }\right\} \\
& +\left[2 e A_{\mu}+\frac{g}{c_{W}}\left(2-Y-2 s_{W}^{2}\right) Z_{\mu}\right]\left[2 e A^{\mu}+\frac{g}{c_{W}}\left(2-Y-2 s_{W}^{2}\right) Z^{\mu}\right] \mathrm{H}^{++} \mathrm{H}^{--} .
\end{aligned}
$$

The first two lines describe the $s$-channel exchange of gauge bosons [82]; whereas all of them enter in the calculation of the vector-boson fusion (VBF) contribution (see next section). These couplings depend on the type of multiplet, i.e., on $T$ and $Y$, the doublycharged scalar belongs to, as do the corresponding cross-sections. In eq. (3.3) we have used eq. (2.1) but omitting superindices for easy reading. The doubly (2) and singly (1) charges have been also made explicit. In figures (tables) 2 and 3 we gather the Feynman diagrams and rules (couplings) for the scalar multiplets satisfying eq. (2.2), which are discussed below for illustration.

Quartic couplings involving neutral scalars $\mathrm{H}^{0}$, i.e., for $T^{\mathrm{H}} \geq\left|T_{3}^{\mathrm{H}^{++}}-2\right|$, also mediate LNV doubly-charged scalar decays once the LN $=2$ neutral component gets a VEV,

$$
\mathcal{L}^{K} \rightarrow \frac{g^{2}}{2} \sqrt{(T+Y)(T+Y-1)(T-Y+2)(T-Y+1)} W_{\mu}^{-} W^{-\mu} \mathrm{H}^{++}\left\langle\mathrm{H}^{0}\right\rangle .
$$




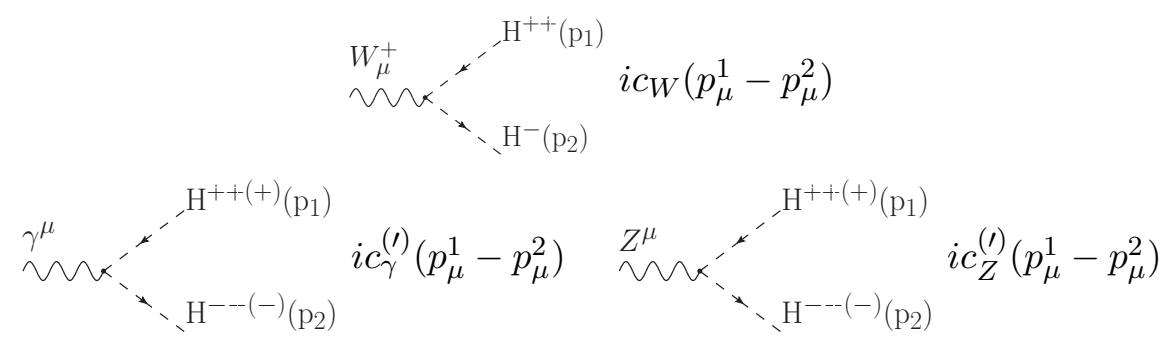

Figure 2. Feynman diagrams and rules for gauge trilinear interactions of doubly $(c)$ and singly $\left(c^{\prime}\right)$ charged scalars. The arrows indicate the LN flow; whereas the $\mathrm{H}^{++(+)}\left(p_{1}\right)$ and $\mathrm{H}^{--(-)}\left(p_{2}\right)$ momenta are leaving the vertex.

\begin{tabular}{|lccccc|}
\hline Vertex & Singlet & Doublet & Triplet & Quadruplet & Quintuplet \\
\hline$c_{W}$ & 0 & $\frac{g}{\sqrt{2}}$ & $g$ & $\sqrt{\frac{3}{2}} g$ & $\sqrt{2} g$ \\
\hline$c_{\gamma}$ & $2 e$ & $2 e$ & $2 e$ & $2 e$ & $2 e$ \\
\hline$c_{Z}$ & $-2 \frac{g}{c_{W}} s_{W}^{2}$ & $\frac{g}{2 c_{W}}\left(1-4 s_{W}^{2}\right)$ & $\frac{g}{c_{W}}\left(1-2 s_{W}^{2}\right)$ & $\frac{g}{2 c_{W}}\left(3-4 s_{W}^{2}\right)$ & $\frac{2 g}{c_{W}}\left(1-s_{W}^{2}\right)$ \\
\hline$c_{\gamma}^{\prime}$ & 0 & $e$ & $e$ & $e$ & $e$ \\
\hline$c_{Z}^{\prime}$ & 0 & $-\frac{g}{2 c_{W}}\left(1+2 s_{W}^{2}\right)$ & $-\frac{g}{c_{W}} s_{W}^{2}$ & $\frac{g}{2 c_{W}}\left(1-2 s_{W}^{2}\right)$ & $\frac{g}{c_{W}}\left(1-s_{W}^{2}\right)$ \\
\hline
\end{tabular}

Table 2. Trilinear Scalar-Scalar-Vector $(\mathcal{S S V})$ couplings for doubly $(c)$ and singly $\left(c^{\prime}\right)$ charged scalars.

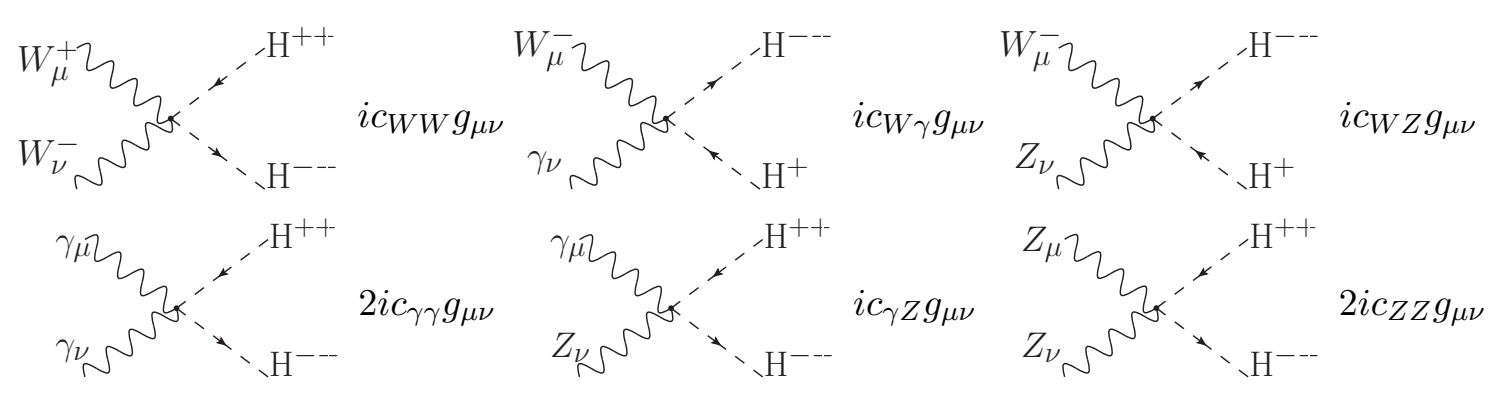

Figure 3. Feynman diagrams and rules for gauge quartic interactions of doubly and singly-charged scalars. The arrows indicate the LN flow.

Multiplets without neutral components can also decay into $W$ pairs by mixing with other multiplets with a neutral component developing a VEV ${ }^{13}$ or through quantum corrections. In order to establish LNV both types of decays $\mathrm{H}^{ \pm \pm} \rightarrow l^{ \pm} l^{ \pm}, W^{ \pm} W^{ \pm}$must be observed. Otherwise, the scalar LN could be just 2 in the former case or 0 in the latter one, but

\footnotetext{
${ }^{13}$ This may be expected in generic ultraviolet completions. As a matter of fact, the effective operators in eq. (2.3) and the effective coupling to $W$ pairs can be obtained from renormalizable theories with further scalars, in particular with a heavy triplet and/or singlet, after integrating them out [29-31].
} 


\begin{tabular}{|lccccc|}
\hline Vertex & Singlet & Doublet & Triplet & Quadruplet & Quintuplet \\
\hline$c_{W W}$ & 0 & $\frac{g^{2}}{2}$ & $g^{2}$ & $\frac{3 g^{2}}{2}$ & $2 g^{2}$ \\
\hline$c_{W \gamma}$ & 0 & $\frac{3}{\sqrt{2}} e g$ & $3 e g$ & $3 \sqrt{\frac{3}{2}} e g$ & $3 \sqrt{2} e g$ \\
\hline$c_{W Z}$ & 0 & $\frac{-3 g^{2} s_{W}^{2}}{\sqrt{2} c_{W}}$ & $\frac{g^{2}}{c_{W}}\left[1-3 s_{W}^{2}\right]$ & $\sqrt{\frac{3}{2}} \frac{g^{2}}{c_{W}}\left[2-3 s_{W}^{2}\right]$ & $\frac{3 \sqrt{2}}{c_{W}} g^{2}\left[1-s_{W}^{2}\right]$ \\
\hline$c_{\gamma \gamma}$ & $4 e^{2}$ & $4 e^{2}$ & $4 e^{2}$ & $4 e^{2}$ & $4 e^{2}$ \\
\hline$c_{\gamma Z}$ & $-8 e^{2} \frac{s_{W}}{c_{W}}$ & $\frac{2 e g}{c_{W}}\left[1-4 s_{W}^{2}\right]$ & $\frac{4 e g}{c_{W}}\left[1-2 s_{W}^{2}\right]$ & $\frac{2 e g}{c_{W}}\left[3-4 s_{W}^{2}\right]$ & $\frac{8 e g}{c_{W}}\left[1-s_{W}^{2}\right]$ \\
\hline$c_{Z Z}$ & $4 g^{2} \frac{s_{W}^{4}}{c_{W}^{2}}$ & $\frac{g^{2}}{4 c_{W}^{2}}\left[1-4 s_{W}^{2}\right]^{2}$ & $\frac{g^{2}}{c_{W}^{2}}\left[1-2 s_{W}^{2}\right]^{2}$ & $\frac{g^{2}}{4 c_{W}^{2}}\left[3-4 s_{W}^{2}\right]^{2}$ & $\frac{4 g^{2}}{c_{W}^{2}}\left[1-s_{W}^{2}\right]^{2}$ \\
\hline
\end{tabular}

Table 3. Quartic Scalar-Scalar-Vector-Vector $(\mathcal{S S V V})$ couplings for VBF doubly-charged pair and associated production.

still conserved. In general, it makes sense to look for decays into lepton as slow as into gauge boson pairs because although the decay into vector bosons is proportional to a VEV which turns out to be minuscule, decays into same-sign charged lepton pairs are stringently constrained by current limits on lepton flavor violation.

\section{Doubly-charged scalar production}

Doubly-charged scalars are pair produced with EW strength through the $s$-channel exchange of photons and $Z$ bosons, $p p \rightarrow \gamma^{*} / Z^{*} \rightarrow \mathrm{H}^{++} \mathrm{H}^{--}$. ${ }^{14}$ Similarly, its associated production with a singly-charged scalar proceeds through $W$ exchange, $p p \rightarrow W^{ \pm *} \rightarrow \mathrm{H}^{ \pm \pm} \mathrm{H}^{ \pm}$. Both cross-sections depend on the quantum numbers of the scalar multiplet the doublycharged scalar belongs to, as do the corresponding couplings in eq. (3.3). In figure 4 we plot them as a function of the doubly-charged scalar mass $m_{\mathrm{H}^{++}}$for the five cases in figure 2 and table 2 and for $\sqrt{s}=8 \mathrm{TeV}$ (the corresponding cross-sections for $\sqrt{s}=14 \mathrm{TeV}$ are shown in $\left.[82]^{15}\right)$.

Both final states can be also produced through VBF but accompanied by two extra jets, $p p \rightarrow \mathrm{H}^{++} \mathrm{H}^{--} j j, \mathrm{H}^{ \pm \pm} \mathrm{H}^{\mp} j j$. These processes are sub-leading as expected from gaugecoupling power counting. The contributing diagrams are depicted in figure 5. Although this mechanism is enhanced because the initial partons are both valence quarks, its size stays below $10 \%$ of the $s$-channel production, being almost negligible for low scalar masses.

\footnotetext{
${ }^{14}$ They can be also singly produced through the effective coupling $\mathrm{H}^{ \pm \pm} W_{\mu}^{\mp} W^{\mu \mp}$ in eq. (3.4). Although, due to the stringent constraints on its size, for instance, implied by the measured value of the $\rho$ parameter (see footnote 12), this production mechanism is in general suppressed to a negligible level, unless a bizarre cancellation is invoked to avoid these bounds [92].

${ }^{15}$ The scalar triplet cross-sections at $7 \mathrm{TeV}$ are plotted, for example, in [60, 61]. For an earlier comparison of the Tevatron and LHC potential see [93].
} 

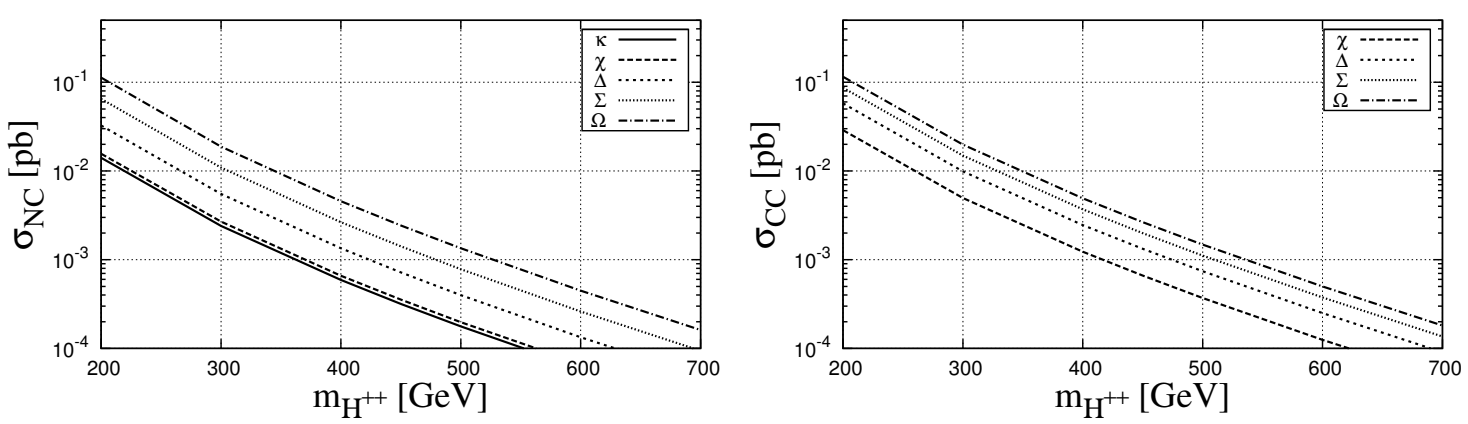

Figure 4. Doubly-charged scalar pair (left) and associated (right) production at the LHC for $\sqrt{s}=8 \mathrm{TeV}$, with scalars $\mathrm{H}$ belonging to a real quintuplet $\Omega$, a quadruplet $\Sigma$, a triplet $\Delta$, a doublet $\chi$ or a singlet $\kappa$ with hypercharges $0,1 / 2,1,3 / 2$ and 2 , respectively.
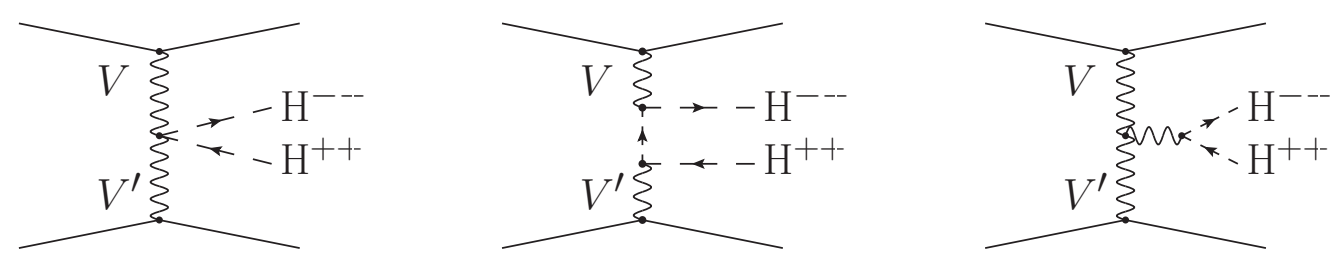

Figure 5. Feynman diagrams contributing to VBF doubly-charged scalar pair production.
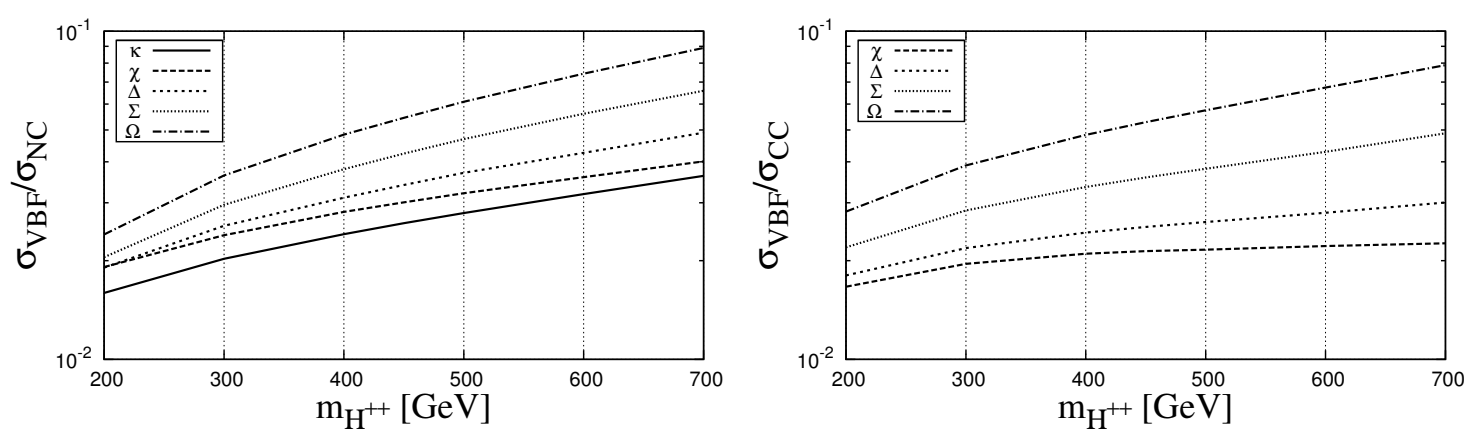

Figure 6. Ratio of the VBF to the pair (left) and associated (right) production cross-sections as a function of the doubly-charged scalar mass for the same multiplets and energy as in figure 4.

In figure 6 we plot the ratio of the VBF to the $s$-channel production cross-section for the same scalar multiplets as in figure $4 .{ }^{16}$ As can be observed in the figure VBF starts to be important only for large masses, when the valence quark parton distribution functions (PDFs) are relatively larger. Anyway, this production mechanism is always present and should be taken into account, although it is possible to separate the corresponding events by requiring two forward extra jets. (Collinear $\gamma$ production can be calculated using the Weizsaecker-Williams approximation [95-97], giving also similar contributions [94].)

\footnotetext{
${ }^{16}$ The ratio for pair production but including only the VBF of two photons, $\gamma \gamma \rightarrow \mathrm{H}^{++} \mathrm{H}^{--}$, is quite similar as previously shown in [94]. These partonic cross-sections diverge when the photon is emitted collinearly and then are sensitive to the limit on the corresponding partonic transverse momentum. We assume as a conservative value $p_{T}^{j}>10 \mathrm{GeV}$ throughout the paper.
} 
Besides, there can be further NP contributions, although in general further suppressed. For example, effective operators contributing to these processes are suppressed by at least two powers of the cutoff scale $\Lambda$ (see appendix A). As a matter of fact, these contact interactions arise naturally in the context, for instance, of non-minimal Composite Higgs Models [98-102]. On the other hand, possibly $s$-channel contributions are in addition suppressed by small far off-shell propagators ${ }^{17}$ while $t$-channel contributions are forbidden, since doubly-charged scalars do not have trilinear couplings to a quark pair.

In summary, although there can be a variety of production mechanisms, in general the main production cross-sections are fixed by the scalar multiplet quantum numbers in eq. (3.3); and thus their measurement would allow to determine the total isospin and hypercharge of the scalar multiplet which the doubly-charged scalar belongs to [82-84]. In the signal simulations below we add the $s$-channel as well as the VBF production, multiplying the former by a K-factor equal to 1.25 [103].

\section{Monte Carlo implementation}

In order to extend the searches of doubly-charged scalars to generic models and to perform phenomenological studies allowing for LNV signals, we have implemented the couplings in eqs. (2.5), (3.3) and (3.4) in MadGraph5 [104]. ${ }^{18}$ The explicit expressions of these couplings for the five scalar multiplets of lowest isospin and hypercharge containing a doubly-charged component, $(T, Y)=(0,2),(1 / 2,3 / 2),(1,1),(3 / 2,1 / 2),(2,0)$, have been included in an UFO model by means of FeynRules v1.6 [105]. It can be downloaded from http://cafpe.ugr.es/index.php/pages/other/software. A set of Param Cards for MadGraph5 can be also found there for all scalar masses considered in the simulations in the text. The K-factor for the leading pair and associated $s$-channel production can be as large as $20-30 \%$ [103]. This and the VBF contributions can be added at will, although the latter are only sizable for large doubly-charged scalar masses.

In order to take into account the scalar decays mediated by the $\mathcal{S F \mathcal { F }}$ and $\mathcal{S} \mathcal{V} \mathcal{V}$ interactions in eqs. (2.5) and (3.4), respectively, it is enough to implement the triplet decay. This is so because all those decays can be mimicked by the triplet one, being only required an additional rescaling of the corresponding rate. In particular, as chirality does not play any role in the subsequent analyses, ${ }^{19}$ we only need to consider $L L$ interactions, i.e., $\Delta^{++} \overline{l_{L i}^{c}} l_{L j}^{\prime}$ and $\Delta^{+} \overline{l_{L i}^{c}} \nu_{L j}^{\prime}$, with the six possible lepton combinations $e e, e \mu, e \tau, \mu \mu, \mu \tau$ and $\tau \tau$ in the doubly-charged case and only three $e \nu_{e}, \mu \nu_{\mu}$ and $\tau \nu_{\tau}$ in the singly-charged one, for neutrinos manifest as missing energy in the detector and hence cannot be distinguished. A new parameter myyuk has been introduced to account for the Yukawa couplings. The scalar decays into $W$ pairs mediated by the interactions $\Delta^{ \pm \pm} W^{ \pm} W^{ \pm}$and $\Delta^{ \pm} W^{ \pm} Z^{0}$ also require the introduction of a new parameter, which we name myvev, proportional to the

\footnotetext{
${ }^{17}$ This is also the case for the Higgs $s$-channel exchange, whose rate is much smaller than the one from the exchange of gauge bosons, unless the effective Higgs coupling to doubly-charged scalars is unnaturally large (equal to $\lambda v$ with $\lambda$ much larger than one).

${ }^{18}$ Further details for its use are given in appendix B.

${ }^{19}$ The helicity of the final leptons can not be measured, except eventually for the tau lepton [106].
} 
corresponding (LNV) VEV. These two parameters have been fixed to values which make the branching ratios into leptons and into bosons of similar magnitude. This guarantees that the total cross-section never vanishes, avoiding numerical problems. These values also imply a narrow scalar width, and hence the width measured from the invariant-mass distribution is dominated by reconstruction effects. Allowing for scalar decays into lepton and boson pairs, non-trivial limits on LNV, not considered yet in experimental searches, can be obtained. We will focus on pair and associated production with one scalar decaying into lepton and the other into boson pairs. ${ }^{20}$ Although no analysis has been designed to look for these specific LNV processes, current searches for doubly-charged resonances decaying only into same-sign charged lepton pairs are already sensitive to this channel. Given that no event excess has been observed, they can be also used to set the first bounds on LNV scalars. We discuss them in detail later.

MadGraph5 parton level events are passed through Pythia v6 [109] to include initial and final state radiation, as well as fragmentation and hadronization, and through Delphes v3 [110] for fast-detector simulation. Jets are reconstructed using an anti-kt algorithm with FastJet v3 [111]. Finally, MadAnalysis v5 [112] is used to perform the analyses. The full sequence of software for Monte Carlo simulation, which has been extensively tested, is available to generalize our results.

\section{Current analyses}

CMS [60] and ATLAS [61] have provided limits on doubly-charged scalars decaying into same-sign $e$ and $\mu$ pairs using samples with four and three isolated charged leptons, as no event excess has been observed. In this section we reproduce their results at $\sqrt{s}=7 \mathrm{TeV}$ using Monte Carlo simulations in order to test our codes. We mimic the detailed analysis by CMS in [60], which besides includes doubly-charged scalar decays into $\tau$ leptons. We then estimate the expected bounds for $8 \mathrm{TeV}$ and an integrated luminosity of $20 \mathrm{fb}^{-1}$ also assuming that no event excess is observed. In this analysis we apply the same cuts and efficiencies as for $7 \mathrm{TeV}$, although the LHC collaborations will certainly optimize both and will provide better limits based on real data. However, no large differences should be expected. In the next section and as another application, we extend these analyses to obtain the corresponding limits on the LNV processes $p p \rightarrow \mathrm{H}^{ \pm \pm} \mathrm{H}^{\mp \mp} \rightarrow \ell^{ \pm} \ell^{ \pm} W^{\mp} W^{\mp}$ and $p p \rightarrow \mathrm{H}^{ \pm \pm} \mathrm{H}^{\mp} \rightarrow \ell^{ \pm} \ell^{ \pm} W^{\mp} Z$, also estimating for both processes the bounds which shall be eventually obtained by the LHC experiments after the next run at $14 \mathrm{TeV}$.

In order to compare with data, the SM backgrounds must be also included. Since the signal efficiencies for $\sqrt{s}=7$ and $8 \mathrm{TeV}$ are similar (we find differences of at most $\sim 10 \%$ ), we assume that this is also the case for the backgrounds and estimate them at $8 \mathrm{TeV}$ scaling the CMS values in table 5 in [60] by a factor of

$$
\frac{\sigma_{8}}{\sigma_{7}} \times \frac{\mathcal{L}_{8}}{\mathcal{L}_{7}} \approx 1.2 \times 4.08
$$

\footnotetext{
${ }^{20}$ There is a different and much more involved way to look for LNV: performing the usual four lepton analyses and also to search for four vector bosons compatible with the same resonance production. This second final state, however, can be only disentangled from the background for very low masses [107, 108].
} 


\begin{tabular}{|lrrr|}
\hline Process & $\sigma[7 \mathrm{TeV}] @ \mathrm{NLO}(\mathrm{pb})$ & $\sigma[8 \mathrm{TeV}] @ \mathrm{NLO}(\mathrm{pb})$ & $\sigma[14 \mathrm{TeV}] @ \mathrm{NLO}(\mathrm{pb})$ \\
\hline Drell-Yan & $(21 \pm 1) \times 10^{2}$ & $(25 \pm 2) \times 10^{2}$ & $(48 \pm 4) \times 10^{2}$ \\
\hline$W^{+} W^{-}$ & $41 \pm 1$ & $50 \pm 2$ & $107 \pm 4$ \\
\hline$W^{ \pm} Z$ & $17 \pm 1$ & $21 \pm 1$ & $47 \pm 2$ \\
\hline$Z Z$ & $5.5 \pm 0.2$ & $6.6 \pm 0.2$ & $14.5 \pm 0.4$ \\
\hline$t \bar{t}$ & $123 \pm 15$ & $176 \pm 22$ & $475 \pm 9$ \\
\hline
\end{tabular}

Table 4. Cross-sections for the main backgrounds considered in the analyses, computed at the NLO in QCD. At the parton level, events have been generated using aMC@NLO [113] with the cut $p_{T}^{j}>10 \mathrm{GeV}$ (in addition and only for Drell-Yan, $l^{+} l^{-}$, we require $p_{T}^{l}>20 \mathrm{GeV}, m_{l^{+} l^{-}}>30 \mathrm{GeV}$ and $\left.\Delta R_{l^{+} l^{-}}>0.4\right)$. The 5 flavor scheme has been used, and the partonic events linked to Pythia by means of the MC@NLO method [114], with the subsequent decay of the $t \bar{t}$ and di-boson final states into their different decay products.

where the first figure is the average of the ratios of the corresponding cross-sections for the largest backgrounds in table 4, Drell-Yan, $W^{+} W^{-}, W^{ \pm} Z, Z Z$ and $t \bar{t}$ production, and the second one is the luminosity ratio 20/4.9. The number of observed events is assumed to be equal to the number of expected background events. (We assume the same at $7 \mathrm{TeV}$ for the scalar masses not gathered in table 5 in [60], taking also in this case the number of expected background events to be equal to the number of events predicted by the SM. ${ }^{21}$ ) For the LHC run at $14 \mathrm{TeV}$ we have instead simulated the complete set of backgrounds in table 4 for an integrated luminosity of $100 \mathrm{fb}^{-1}$, assuming again that the observed number of events coincides with the expected number of background events after cuts.

CMS has performed six different analyses using four and three isolated charged lepton samples, $\ell \ell \ell \ell$ and $\ell \ell \ell$, with $\ell=e, \mu$ and $\tau_{h}$ (although at least two of them must be same-sign electrons or muons). In the first three studies doubly-charged scalars are pair produced and assumed to decay $100 \%$ of the time into $\ell^{ \pm} \ell^{ \pm}, \ell^{ \pm} \tau^{ \pm}$and $\tau^{ \pm} \tau^{ \pm}$in turn. The cuts and efficiencies are optimized for each case, and events are generated for different scalar masses. In table 5 we collect the corresponding cuts and our estimates of the cumulative efficiencies cut-by-cut for a low $(200 \mathrm{GeV})$ and a relatively large $(500 \mathrm{GeV})$ scalar mass for illustration. As pointed out by CMS, the efficiencies slightly increase with the scalar mass. On the other hand, the mass window is the most effective cut, implying a large reduction of the background. In the $\ell^{ \pm} \tau^{ \pm} \ell^{\mp} \tau^{\mp}$ and $\tau^{ \pm} \tau^{ \pm} \tau^{\mp} \tau^{\mp}$ analyses tau decays into hadrons are also taking into account. Hadronic tau leptons $\left(\tau_{h}\right)$ are tagged by a pure geometrical

\footnotetext{
${ }^{21}$ We assume that the number of events for $m_{\mathrm{H}^{ \pm \pm}}=500 \mathrm{GeV}$ is the same as for $450 \mathrm{GeV}$ in [60], and no background events are expected and none is observed after the corresponding selection cuts for $m_{\mathrm{H}^{ \pm \pm}}=$ 600 and $700 \mathrm{GeV}$.
} 
method in Delphes, becoming a jet a potential $\tau_{h}$ if a generated $\tau$ is found within a fixed distance $\Delta R$ of the jet axis.

Analogously, in table 6 we gather the corresponding cuts and estimated cumulative efficiencies for the three charged lepton sample and doubly-charged scalar associated production. Similarly to the doubly-charged scalar, the singly-charged scalar $\mathrm{H}^{ \pm}$is assumed to decay $100 \%$ of the time into $\ell^{ \pm} \nu_{\ell}, \ell^{ \pm} \nu_{\tau}\left(\tau^{ \pm} \nu_{\ell}\right)$ and $\tau^{ \pm} \nu_{\tau}$ in turn. In order to be conservative we make use of the $\ell^{ \pm} \tau^{ \pm} \tau^{\mp} \nu_{\ell}$ efficiency for $\ell^{ \pm} \tau^{ \pm} \ell^{\mp} \nu_{\tau}$, too, although the former is smaller due to the required extra tau branching ratio into electrons and muons. As emphasized in ref. [60], the efficiencies for the three-lepton analyses are near a factor 2 smaller than for the corresponding four-lepton analyses.

Using the estimated efficiencies for seven doubly-charged scalar masses, $m_{\mathrm{H}^{ \pm \pm}}=200$, $300,400,450,500,600$ and $700 \mathrm{GeV},{ }^{22}$ and the expected background and observed number of events, we can draw the corresponding exclusion plots as no event excess has been observed. In figure 7 from top to bottom we plot the 95\% C.L. limits (see appendix C for the pertinent definitions) for the analyses in tables 5 (left) and 6 (right). ${ }^{23}$ The bounds very much coincide with those reported by CMS for a doubly-charged scalar mediating the seesaw of type II, ranging from 400 to $200 \mathrm{GeV}$ depending on the scalar decay mode. (What in particular implies that the efficiencies we use are consistent within the fast simulation algorithm uncertainties with those obtained by CMS.) However, if the doubly-charged scalar belongs to other type of multiplet, its cross-sections vary and so the bounds on its mass. We superimpose in the plots the corresponding predictions for the five multiplets discussed before, increasing the limits with the cross-section (total isospin). The most stringent bounds are then obtained for the quintuplet, being typically $\sim 150 \mathrm{GeV}$ higher than for the triplet (see-saw of type II).

Similarly, we can estimate the limits which may be obtained at $\sqrt{s}=8 \mathrm{TeV}$ for an integrated luminosity of $20 \mathrm{fb}^{-1}$, assuming that no departure from the SM is observed. The efficiencies calculated at $8 \mathrm{TeV}$ for the six decay modes and the same CMS cuts are very similar to those at $7 \mathrm{TeV}$ for the seven scalar masses. In figure 8 we show the corresponding exclusion plots. ${ }^{24}$ Typically, all bounds are around $\sim 100 \mathrm{GeV}$ higher.

\section{Final state-dependent efficiencies and LNV bounds at the LHC}

A convenient way of giving a more complete information on the experimental bounds on $\mathrm{NP}$ is also providing the full set of efficiencies for the different processes considered. In this way the limits on new models can be in general estimated without performing new analyses. For instance, in the case at hand, just giving the bounds on the processes with the doubly-charged scalars decaying $100 \%$ of the time into $\ell^{ \pm} \ell^{ \pm}\left(p p \rightarrow \mathrm{H}^{ \pm \pm} \mathrm{H}^{\mp \mp} \rightarrow \ell^{ \pm} \ell^{ \pm} \ell^{\mp} \ell^{\mp}\right)$ and into $\ell^{ \pm} \tau^{ \pm}\left(p p \rightarrow \mathrm{H}^{ \pm \pm} \mathrm{H}^{\mp \mp} \rightarrow \ell^{ \pm} \tau^{ \pm} \ell^{\mp} \tau^{\mp}\right)$, in turn, one can estimate the corresponding limits on a model where the doubly-charged scalars decay half of the time into each of these

\footnotetext{
${ }^{22}$ Efficiencies for intermediate masses can be obtained by interpolation.

${ }^{23}$ Exclusion limits for intermediate masses are obtained by splines interpolation.

${ }^{24}$ The number of background and observed events at $8 \mathrm{TeV}$ is estimated scaling the $7 \mathrm{TeV}$ values with eq. (6.1). Exclusion limits for intermediate masses are obtained by splines interpolation.
} 


\begin{tabular}{|c|c|c|c|}
\hline \multicolumn{2}{|r|}{ Cuts } & \multicolumn{2}{|c|}{$\begin{array}{c}\text { Efficiencies } \\
m_{\mathrm{H}^{ \pm \pm}}=200 \mathrm{GeV} \quad 500 \mathrm{GeV}\end{array}$} \\
\hline \multicolumn{4}{|c|}{$\ell^{ \pm} \ell^{ \pm} \ell^{\mp} \ell^{\mp}$} \\
\hline Basic cuts & $p_{T}^{\ell_{1(2)}}>20(10) \mathrm{GeV},\left|\eta^{\ell}\right|<2.5$ & 68 & 72 \\
\hline Total $p_{T}$ & $\sum p_{T}^{\ell}>0.6 m_{\mathrm{H}^{ \pm \pm}}+130 \mathrm{GeV}$ & 99 & 100 \\
\hline Mass window & $m_{\ell^{ \pm} \ell^{ \pm}} \in\left[0.9 m_{\mathrm{H}^{ \pm \pm}}, 1.1 m_{\mathrm{H}^{ \pm \pm}}\right]$ & 92 & 89 \\
\hline Total & & 62 & 64 \\
\hline \multicolumn{4}{|c|}{$\ell^{ \pm} \tau^{ \pm} \ell^{\mp} \tau^{\mp}$} \\
\hline Basic cuts & $p_{T}^{\ell_{1(2)}}>20(10) \mathrm{GeV},\left|\eta^{\ell}\right|<2.5$ & 16 & 23 \\
\hline Total $p_{T}$ & $\sum p_{T}^{\ell}>m_{\mathrm{H}^{ \pm \pm}}+100$ or $>400 \mathrm{GeV}$ & 82 & 99 \\
\hline$Z$ veto & $\left|m_{\ell^{ \pm} \ell^{ \pm}}-m_{Z}\right|>10 \mathrm{GeV}$ & 85 & 92 \\
\hline Mass window & $m_{\ell^{ \pm} \ell^{ \pm}} \in\left[0.5 m_{\mathrm{H}^{ \pm \pm}}, 1.1 m_{\mathrm{H}^{ \pm \pm}}\right]$ & 81 & 66 \\
\hline Total & & 9.0 & 14 \\
\hline \multicolumn{4}{|c|}{$\tau^{ \pm} \tau^{ \pm} \tau^{\mp} \tau^{\mp}$} \\
\hline Basic cuts & $p_{T}^{\ell_{1(2)}}>20(10) \mathrm{GeV},\left|\eta^{\ell}\right|<2.5$ & 3.0 & 5.0 \\
\hline Total $p_{T}$ & $\sum p_{T}^{\ell}>120 \mathrm{GeV}$ & 99 & 100 \\
\hline$Z$ veto & $\left|m_{\ell^{ \pm} \ell^{ \pm}}-m_{Z}\right|>50 \mathrm{GeV}$ & 82 & 86 \\
\hline$\Delta \phi$ & $\Delta \phi_{\ell^{ \pm} \ell^{ \pm}}<2.5$ & 80 & 80 \\
\hline Total & & 2.0 & 3.5 \\
\hline \multicolumn{4}{|c|}{$\ell^{ \pm} \ell^{ \pm} \ell^{\mp} \nu_{\ell}$} \\
\hline Basic cuts & $p_{T}^{\ell_{1(2)}}>20(10) \mathrm{GeV},\left|\eta^{\ell}\right|<2.5$ & 78 & 82 \\
\hline Total $p_{T}$ & $\sum p_{T}^{\ell}>1.1 m_{\mathrm{H}^{ \pm \pm}}+60 \mathrm{GeV}$ & 84 & 87 \\
\hline$Z$ veto & $\left|m_{\ell^{ \pm} \ell^{ \pm}}-m_{Z}\right|>80 \mathrm{GeV}$ & 59 & 90 \\
\hline$\Delta \phi$ & $\Delta \phi_{\ell^{ \pm} \ell^{ \pm}}<m_{\mathrm{H}^{ \pm \pm}}(\mathrm{GeV}) / 600+1.95$ & 86 & 94 \\
\hline Mass window & $m_{\ell^{ \pm} \ell^{ \pm}} \in\left[0.9 m_{\mathrm{H}^{ \pm \pm}}, 1.1 m_{\mathrm{H}^{ \pm \pm}}\right]$ & 94 & 93 \\
\hline Total & & 31 & 56 \\
\hline Expected background & & 0.99 & 0.14 \\
\hline Observed events & & 2 & 1 \\
\hline
\end{tabular}




\begin{tabular}{|llcr|}
\hline \multicolumn{1}{|c}{ Cuts } & \multicolumn{2}{c|}{ Efficiencies } \\
& \multicolumn{1}{c|}{$\ell^{ \pm} \tau^{ \pm} \ell^{\mp} \nu_{\tau}\left(\tau^{\mp} \nu_{\ell}\right)$} & $m_{H}=200 \mathrm{GeV}$ & $500 \mathrm{GeV}$ \\
\hline \multicolumn{1}{|c|}{$p_{T}^{\ell_{1(2)}}>20(10) \mathrm{GeV},\left|\eta^{\ell}\right|<2.5$} & 16 & 20 \\
\hline Basic cuts & $\sum p_{T}^{\ell}>0.85 m_{\mathrm{H}^{ \pm \pm}}+125 \mathrm{GeV}$ & 38 & 48 \\
Total $p_{T}$ & $\left|m_{\ell^{ \pm} \ell^{ \pm}}-m_{Z}\right|>80 \mathrm{GeV}$ & 85 & 93 \\
$Z$ veto & $E_{T}^{\text {miss }}>20 \mathrm{GeV}$ & 98 & 99 \\
$E_{T}^{\text {miss }}$ & $\Delta \phi_{\ell^{ \pm} \ell^{ \pm}}<m_{\mathrm{H}^{ \pm \pm}}(\mathrm{GeV}) / 200+1.15$ & 83 & 100 \\
$\Delta \phi$ & $m_{\ell^{ \pm} \ell^{ \pm}} \in\left[0.5 m_{\mathrm{H}^{ \pm \pm}}, 1.1 m_{\mathrm{H}^{ \pm \pm}}\right]$ & 91 & 89 \\
Mass window & & 3.8 & 7.9 \\
\hline Total & & 1.51 & 0.18 \\
\hline Expected background & & 3 & 1 \\
Observed events & & & \\
\hline
\end{tabular}

Table 5. Applied cuts to the four isolated charged lepton sample $\ell \ell \ell$, with two $\ell=e$ or $\mu$ and the other two $e, \mu$ or $\tau_{h}$, and efficiency percentage for each successive cut for the final states $\ell^{ \pm} \ell^{ \pm} \ell^{\mp} \ell^{\mp}$, $\ell^{ \pm} \tau^{ \pm} \ell^{\mp} \tau^{\mp}$ and $\tau^{ \pm} \tau^{ \pm} \tau^{\mp} \tau^{\mp}$ and two representative scalar masses. The basic transverse momentum cuts are imposed on the two leptons, electrons or muons, required by the trigger; whereas the transverse momentum sum is over the four charged leptons, as the generic pseudo-rapidity cut. In the three analyses no background events are expected and no event is observed for an integrated luminosity of $4.9 \mathrm{fb}^{-1}$ at $\sqrt{s}=7 \mathrm{TeV}$.

two final states, but without being able to use the $\ell^{ \pm} \ell^{ \pm} \ell^{\mp} \tau^{\mp}$ events and hence half of the statistics. With this in mind, we collect the efficiencies for the four-lepton and three-lepton analyses in tables 7 and 8 , respectively, for all two-body decays of the doubly and singlycharged scalars, $\ell \ell, \ell \tau, \tau \tau, W W$ and $\ell \nu, \tau \nu, W Z$, and seven scalar masses, $m_{\mathrm{H}^{ \pm \pm}}=200,300$, 400, 450, 500, 600 and $700 \mathrm{GeV}$. As $\mathrm{H}^{ \pm \pm}\left(\mathrm{H}^{ \pm}\right)$has 4 (3) different two-body decay modes, there are a priori $4 \times 4+4 \times 3=28$ final states and hence $\epsilon_{i j}$ efficiencies. But for pair production $\epsilon_{i j}=\epsilon_{j i}$, being then only 10 of the 16 efficiencies independent. In table 7 we omit the $\mathrm{H}^{ \pm \pm} \mathrm{H}^{\mp}$ decay modes because all their efficiencies are below $\sim 0.1 \%$. For both analyses, the applied cuts are common to all final states, thus not optimizing the different modes but the full set. Following CMS analyses for benchmark points we choose the cuts for $\ell^{ \pm} \tau^{ \pm} \ell^{\mp} \tau^{\mp}$ in table 5 and for $\ell^{ \pm} \tau^{ \pm} \ell^{\mp} \nu_{\tau}\left(\tau^{\mp} \nu_{\ell}\right)$ in table 6 to calculate the efficiencies in tables 7 and 8 , respectively. They grow with the scalar mass because the cuts stay fixed. For example, electrons and muons are harder for larger scalar masses and hence they satisfy more easily not only the basic cuts but the cuts on $\sum p_{T}^{\ell}$ and on $E_{T}^{\text {miss }}$. The latter is particularly stringent for pair produced events because in this case the missing energy comes either from missed leptons or from missing energy measurement errors and hence, 


\begin{tabular}{|llrr|}
\hline \multicolumn{4}{|c|}{$\tau^{ \pm} \tau^{ \pm} \tau^{\mp} \nu_{\tau}$} \\
\hline Basic cuts & $p_{T}^{\ell_{1(2)}}>20(10) \mathrm{GeV},\left|\eta^{\ell}\right|<2.5$ & 4.2 & 8.3 \\
Total $p_{T}$ & $\sum p_{T}^{\ell}>m_{\mathrm{H}^{ \pm \pm}}-10 \mathrm{or}>200 \mathrm{GeV}$ & 55 & 91 \\
$Z$ veto & $\left|m_{\ell^{ \pm} \ell^{ \pm}}-m_{Z}\right|>50 \mathrm{GeV}$ & 80 & 85 \\
$E_{T}^{\text {miss }}$ & $E_{T}^{\text {miss }}>40 \mathrm{GeV}$ & 86 & 97 \\
$\Delta \phi$ & $\Delta \phi_{\ell^{ \pm} \ell^{ \pm}}<2.1$ & 84 & 84 \\
Mass window & $m_{\ell^{ \pm} \ell^{ \pm}} \in\left[0.5 m_{\mathrm{H}^{ \pm \pm}}-20 \mathrm{GeV}, 1.1 m_{\mathrm{H}^{ \pm \pm}}\right]$ & 76 & 42 \\
\hline Total & & 1.0 & 2.2 \\
\hline Expected background & 1.51 & 0.18 \\
Observed events & 3 & 1 \\
\hline
\end{tabular}

Table 6. Applied cuts to the three isolated charged lepton sample $\ell \ell \ell$, with two $\ell=e$ or $\mu$ and the third one $e, \mu$ or $\tau_{h}$, and efficiency percentage for each successive cut for the final states $\ell^{ \pm} \ell^{ \pm} \ell^{\mp} \nu_{\ell}$, $\ell^{ \pm} \tau^{ \pm} \ell^{\mp} \nu_{\tau}\left(\tau^{\mp} \nu_{\ell}\right)$ and $\tau^{ \pm} \tau^{ \pm} \tau^{\mp} \nu_{\tau}$ and two representative scalar masses. The basic transverse momentum cuts are imposed on the two leptons, electrons or muons, required by the trigger; whereas the transverse momentum sum is over the three charged leptons, as the generic pseudo-rapidity cut. The expected background events as well as the observed ones for an integrated luminosity of $4.9 \mathrm{fb}^{-1}$ at $\sqrt{s}=7 \mathrm{TeV}$ are also listed.

\begin{tabular}{|lrrrrrrr|}
\hline$\epsilon_{i j}^{(4 \ell \ell}$ & $m_{\mathrm{H}^{ \pm \pm}}=200$ & 300 & 400 & 450 & 500 & 600 & 700 \\
\hline$\ell \ell \ell \ell$ & 53 & 62 & 67 & 68 & 69 & 70 & 71 \\
\hline$\ell \ell \ell \tau$ & 23 & 27 & 30 & 31 & 32 & 32 & 33 \\
\hline$\ell \ell \tau \tau$ & 7.2 & 8.7 & 9.4 & 9.4 & 9.5 & 9.7 & 9.9 \\
\hline$\ell \ell W W$ & 0.6 & 1.1 & 1.3 & 1.3 & 1.4 & 1.4 & 1.5 \\
\hline$\ell \tau \ell \tau$ & 9.0 & 11 & 13 & 14 & 14 & 14 & 14 \\
\hline$\ell \tau \tau \tau$ & 2.0 & 2.6 & 3.3 & 3.4 & 3.4 & 3.4 & 3.7 \\
\hline$\ell \tau W W$ & 0.2 & 0.4 & 0.6 & 0.6 & 0.6 & 0.7 & 0.7 \\
\hline$\tau \tau \tau \tau$ & 0.3 & 0.5 & 0.6 & 0.7 & 0.7 & 0.7 & 0.7 \\
\hline$\tau \tau W W$ & 0.1 & 0.1 & 0.2 & 0.2 & 0.2 & 0.2 & 0.2 \\
\hline$W W W W$ & $<0.1$ & $<0.1$ & $<0.1$ & $<0.1$ & $<0.1$ & $<0.1$ & $<0.1$ \\
\hline
\end{tabular}

Table 7. Efficiency percentages $\epsilon_{i j}^{(4 \ell)}$ for different scalar masses (in $\mathrm{GeV}$ ) and final modes $i j$ for four-lepton analyses at $\sqrt{s}=7 \mathrm{TeV}$ and the $\ell^{ \pm} \tau^{ \pm} \ell^{\mp} \tau^{\mp}$ cuts in table 5 . We omit the efficiencies for associated production processes because all of them are below $\sim 0.1 \%$, as these final states do not pass the cuts imposed on the four-lepton sample. 


\begin{tabular}{|c|c|c|c|c|c|c|c|}
\hline$\epsilon_{i j}^{(3 \ell)}$ & $m_{\mathrm{H}^{ \pm \pm}}=200$ & 300 & 400 & 450 & 500 & 600 & 700 \\
\hline elel & 2.7 & 5.0 & 7.5 & 8.7 & 9.5 & 10 & 11 \\
\hline$\ell \ell \ell \tau$ & 17 & 25 & 31 & 33 & 34 & 34 & 35 \\
\hline$\ell \ell \tau \tau$ & 18 & 24 & 28 & 29 & 30 & 31 & 32 \\
\hline$\ell \ell W W$ & 6.9 & 13 & 17 & 18 & 19 & 20 & 21 \\
\hline$\ell \tau \ell \tau$ & 14 & 19 & 24 & 24 & 25 & 26 & 26 \\
\hline$\ell \tau \tau \tau$ & 4.9 & 6.9 & 8.6 & 8.6 & 9.0 & 9.2 & 9.3 \\
\hline$\ell \tau W W$ & 2.3 & 4.6 & 6.3 & 6.6 & 6.7 & 7.0 & 7.2 \\
\hline$\tau \tau \tau \tau$ & 0.8 & 1.2 & 1.4 & 1.4 & 1.4 & 1.6 & 1.6 \\
\hline$\tau \tau W W$ & 0.4 & 0.8 & 1.0 & 1.0 & 1.1 & 1.2 & 1.2 \\
\hline$W W W W$ & $<0.1$ & 0.3 & 0.4 & 0.4 & 0.5 & 0.5 & 0.5 \\
\hline$\ell \ell \ell \nu$ & 38 & 53 & 64 & 66 & 68 & 70 & 72 \\
\hline$\ell \ell \tau \nu$ & 18 & 26 & 31 & 33 & 34 & 35 & 36 \\
\hline$\ell \ell W Z$ & 5.0 & 8.5 & 11 & 12 & 13 & 13 & 14 \\
\hline$\ell \tau \ell \nu$ & 15 & 21 & 26 & 27 & 28 & 29 & 29 \\
\hline$\ell \tau \tau \nu$ & 3.8 & 5.4 & 7.4 & 7.6 & 7.9 & 8.4 & 8.5 \\
\hline$\ell \tau W Z$ & 1.6 & 2.9 & 3.9 & 4.0 & 4.1 & 4.5 & 4.5 \\
\hline$\tau \tau \ell \nu$ & 2.3 & 3.4 & 4.3 & 4.5 & 4.6 & 4.8 & 4.9 \\
\hline$\tau \tau \tau \nu$ & 0.4 & 0.6 & 0.7 & 0.8 & 0.8 & 0.8 & 0.9 \\
\hline$\tau \tau W Z$ & 0.2 & 0.4 & 0.6 & 0.6 & 0.6 & 0.6 & 0.7 \\
\hline$W W \ell \nu$ & 0.3 & 0.7 & 1.0 & 1.0 & 1.0 & 1.1 & 1.2 \\
\hline$W W \tau \nu$ & 0.1 & 0.2 & 0.3 & 0.4 & 0.4 & 0.4 & 0.4 \\
\hline$W W W Z$ & $<0.1$ & 0.1 & 0.1 & 0.2 & 0.2 & 0.2 & 0.2 \\
\hline
\end{tabular}

Table 8. Efficiency percentages $\epsilon_{i j}^{(3 \ell)}$ for different scalar masses (in GeV) and final modes $i j$ for three-lepton analyses at $\sqrt{s}=7 \mathrm{TeV}$ and the $\ell^{ \pm} \tau^{ \pm} \ell^{\mp} \nu_{\tau}\left(\tau^{\mp} \nu_{\ell}\right)$ cuts in table 6 . 

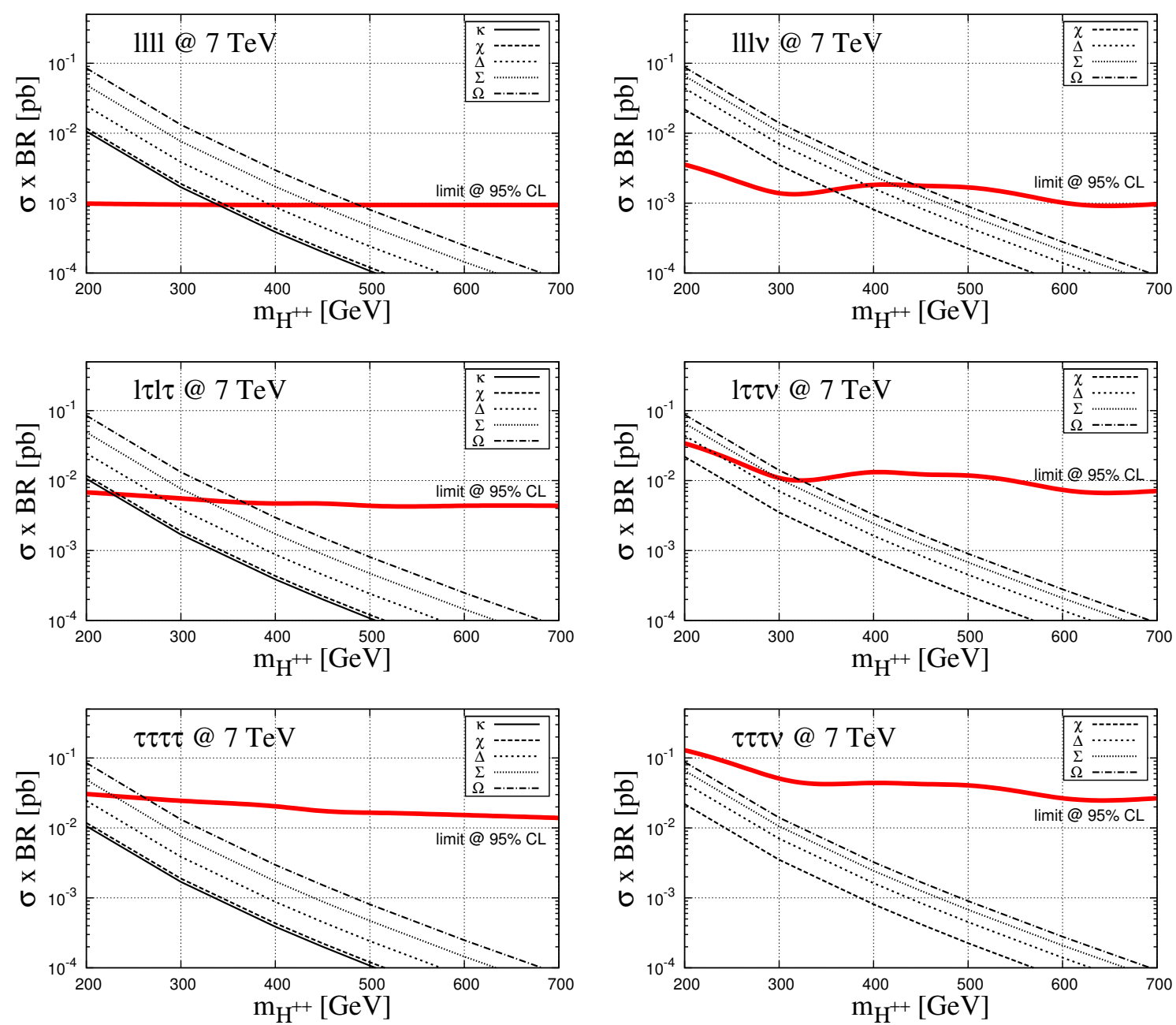

Figure 7. Estimated $95 \%$ C.L. limits on the final modes $\ell^{ \pm} \ell^{ \pm} \ell^{\mp} \ell^{\mp}, \ell^{ \pm} \tau^{ \pm} \ell^{\mp} \tau^{\mp}, \tau^{ \pm} \tau^{ \pm} \tau^{\mp} \tau^{\mp}$ (left column from top to bottom) and $\ell^{ \pm} \ell^{ \pm} \ell^{\mp} \nu_{\ell}, \ell^{ \pm} \tau^{ \pm} \ell^{\mp} \nu_{\ell}\left(\tau^{\mp} \nu_{\tau}\right), \tau^{ \pm} \tau^{ \pm} \tau^{\mp} \nu_{\tau}$ (right column from top to bottom) as a function of the doubly-charged scalar mass $\mathrm{H}^{++}$for $\sqrt{s}=7 \mathrm{TeV}$ and $\mathcal{L}_{\text {int }}=4.9 \mathrm{fb}^{-1}$ at LHC. There are superimposed the corresponding cross-sections for the five scalar multiplets of lowest isospin and hypercharge containing a doubly-charged component, a singlet $\kappa$, a doublet $\chi$, a triplet $\Delta$, a quadruplet $\Sigma$ and a quintuplet $\Omega$.

it is relatively small. In general the missing energy and the total transverse momentum in the event are correlated, too. Whereas the $Z$ veto is also less restrictive for larger masses, in contrast with the mass window constraint for events involving tau leptons. Changes on parton shower and detector simulation inputs stand for variations in the efficiencies of around $\sim 15 \%$. This is the total uncertainty which we assign to the estimates in tables 7 and 8. They agree with the efficiencies quoted in [60] when comparison is possible.

No dedicated searches for LNV signals have been performed in doubly-charged scalar production analyses up to now. However, the ATLAS and CMS searches for doublycharged scalars using four and three-lepton samples are also sensitive to LNV final states. As already emphasized, we can make use of the pertinent efficiencies in tables 7 and 8 in 

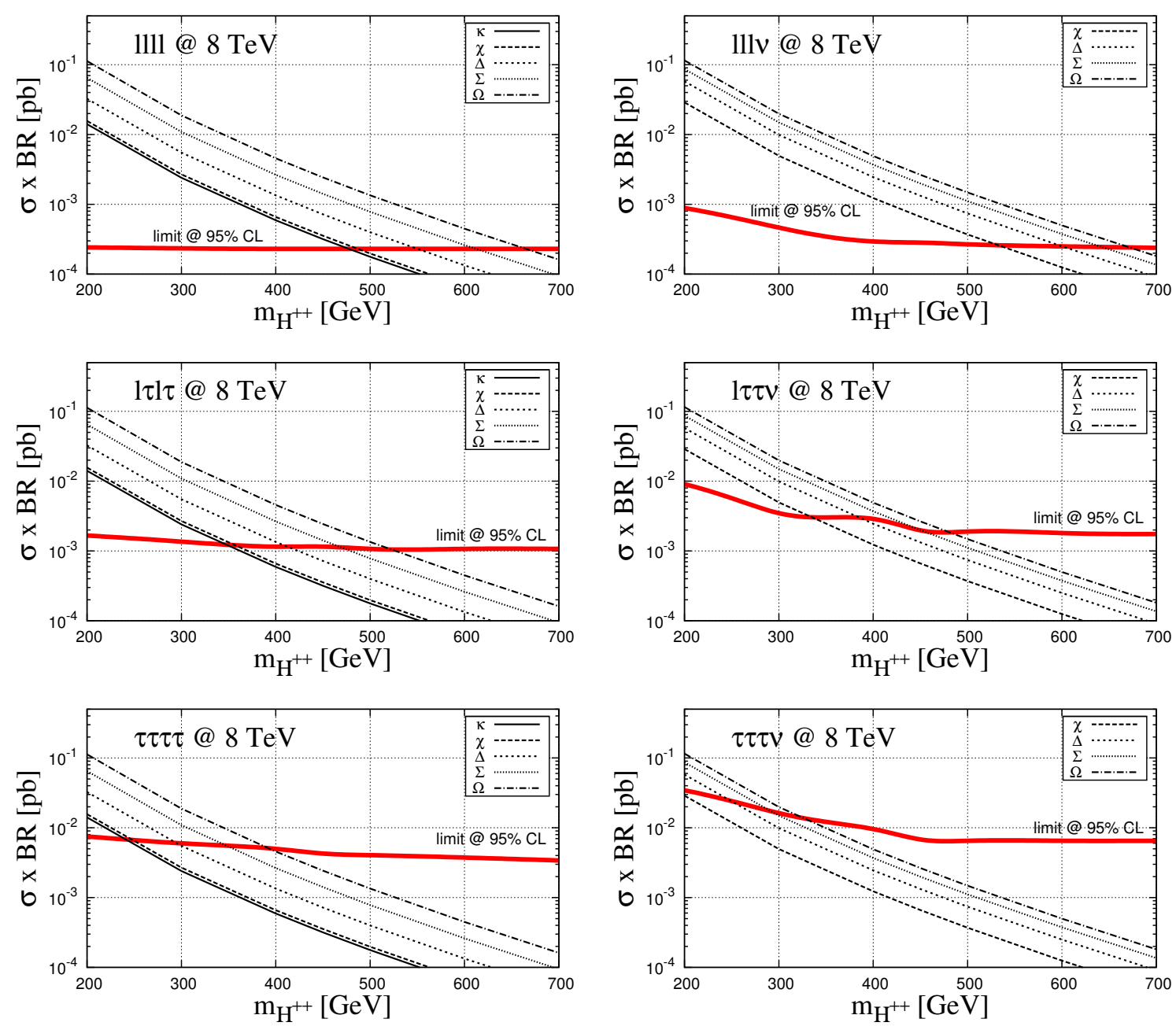

Figure 8. The same as in figure 7 but for $8 \mathrm{TeV}$ and $20 \mathrm{fb}^{-1}$.

order to derive the corresponding bounds, for no event excess has been observed in the four and three-lepton analyses. We restrict ourselves to the LNV final states $\ell \ell W W$ and $\ell \ell W Z$ because they have the largest efficiencies (see tables 7 and 8), showing the results for the three-lepton analysis only for it is by far the most sensitive to LNV. For a given integrated luminosity the number of signal events $N_{\ell \ell W W(\ell \ell W Z)}^{(3 \ell)}$ is estimated multiplying $\mathcal{L}_{\text {int }}$ by the corresponding cross-section, $\sigma_{N C(C C)}$ for doubly-charged scalar pair (associated) production, times the model branching ratios into $\ell \ell$ and $W W(W Z)$ times the selection efficiency:

$$
\begin{aligned}
N_{\ell \ell W W}^{(3 \ell)} & =\mathcal{L}_{\text {int }} \times \sigma_{N C} \times \mathrm{BR}\left(\mathrm{H}^{ \pm \pm} \rightarrow \ell^{ \pm} \ell^{ \pm}\right) \times \mathrm{BR}\left(\mathrm{H}^{\mp \mp} \rightarrow W^{\mp} W^{\mp}\right) \times \epsilon_{\ell \ell W W}^{(3 \ell)}, \\
N_{\ell \ell W Z}^{(3 \ell)} & =\mathcal{L}_{\text {int }} \times \sigma_{C C} \times \mathrm{BR}\left(\mathrm{H}^{ \pm \pm} \rightarrow \ell^{ \pm} \ell^{ \pm}\right) \times \mathrm{BR}\left(\mathrm{H}^{\mp} \rightarrow W^{\mp} Z\right) \times \epsilon_{\ell \ell W Z}^{(3 \ell)} .
\end{aligned}
$$

Making use of these expressions and the number of expected background events and observed events we can derive the exclusion plots for $\sigma_{N C(C C)}$ (see appendix $\mathrm{C}$ ). In figure 9 we plot the corresponding limits assuming that the heavy scalars have the same decay rate 

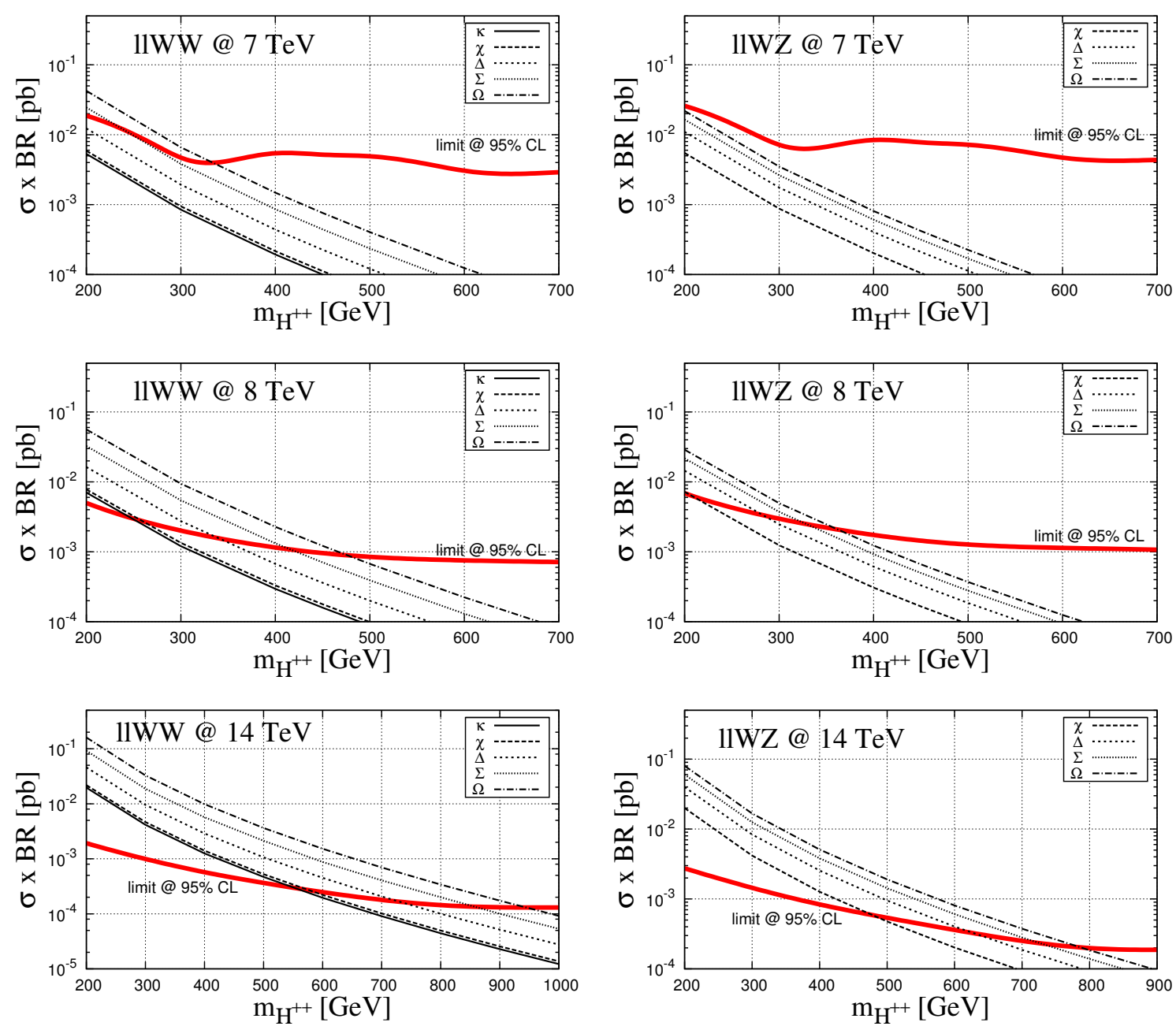

Figure 9. $95 \%$ C.L. limits on the LNV channels $p p \rightarrow \mathrm{H}^{ \pm \pm} \mathrm{H}^{\mp \mp} \rightarrow \ell^{ \pm} \ell^{ \pm} W^{\mp} W^{\mp}$ (left) and $p p \rightarrow \mathrm{H}^{ \pm \pm} \mathrm{H}^{\mp} \rightarrow \ell^{ \pm} \ell^{ \pm} W^{\mp} Z$ (right) as a function of the $\mathrm{H}^{ \pm \pm}$mass for $\sqrt{s}=7,8$ and $14 \mathrm{TeV}$ and $\mathcal{L}_{\text {int }}=4.9,20$ and $100 \mathrm{fb}^{-1}$ at LHC, respectively (from top to bottom). There are superimposed the corresponding cross-sections for the five scalar multiplets of lowest isospin and hypercharge containing a doubly-charged component: the singlet $\kappa$, the doublet $\chi$, the triplet $\Delta$, the quadruplet $\Sigma$ and the quintuplet $\Omega$.

(50\%) into light (first two families) lepton and gauge boson pairs. We superimpose the cross-sections for the different doubly-charged multiplet assignments conveniently normalized by the assumed branching ratios: $1 / 2$ for pair and $1 / 4$ for associated production. The exclusion plots for $\ell \ell W W(\ell \ell W Z)$ are shown on the left (right). From top to bottom we gather the LNV bounds at 7,8 and $14 \mathrm{TeV}$. The number of expected background and observed events for the first two energies are the same as for the $\ell^{ \pm} \tau^{ \pm} \ell^{\mp} \nu_{\tau}\left(\tau^{\mp} \nu_{\ell}\right)$ threelepton analysis in the previous section; whereas for $14 \mathrm{TeV}$ we assume them to be equal, finding for the backgrounds in table 4 with the same cuts a total of $42,37,18,15,9,7$ and 0 events for an integrated luminosity of $100 \mathrm{fb}^{-1}$ and $m_{\mathrm{H}^{ \pm \pm}}=200,300,400,450$, 500, 600 and $700 \mathrm{GeV}$, respectively. In tables 9 and 10 we collect the efficiencies at this 


\begin{tabular}{|lrrrrrrr|}
\hline$\epsilon_{i j}^{(4 \ell)}$ & $m_{\mathrm{H}^{ \pm \pm}}=200$ & 300 & 400 & 450 & 500 & 600 & 700 \\
\hline$\ell \ell \ell \ell$ & 54 & 62 & 66 & 68 & 69 & 69 & 70 \\
\hline$\ell \ell W W$ & 0.6 & 0.9 & 1.1 & 1.4 & 1.4 & 1.4 & 1.5 \\
\hline$W W W W$ & $<0.1$ & $<0.1$ & $<0.1$ & $<0.1$ & $<0.1$ & $<0.1$ & $<0.1$ \\
\hline$\ell \ell \ell \nu$ & $<0.1$ & $<0.1$ & $<0.1$ & $<0.1$ & $<0.1$ & $<0.1$ & $<0.1$ \\
\hline$\ell \ell W Z$ & $<0.1$ & 0.1 & 0.1 & 0.1 & 0.1 & 0.1 & 0.1 \\
\hline$W W \ell \nu$ & $<0.1$ & $<0.1$ & $<0.1$ & $<0.1$ & $<0.1$ & $<0.1$ & $<0.1$ \\
\hline$W W W Z$ & $<0.1$ & $<0.1$ & $<0.1$ & $<0.1$ & $<0.1$ & $<0.1$ & $<0.1$ \\
\hline
\end{tabular}

Table 9. Efficiency percentages $\epsilon_{i j}^{(4 \ell)}$ for different scalar masses (in $\mathrm{GeV}$ ) and final modes $i j$ for four-lepton analyses at $\sqrt{s}=14 \mathrm{TeV}$ and the $\ell^{ \pm} \tau^{ \pm} \ell^{\mp} \tau^{\mp}$ cuts in table 5 .

\begin{tabular}{|c|c|c|c|c|c|c|c|}
\hline$\epsilon_{i j}^{(3 \ell)}$ & $m_{\mathrm{H}^{ \pm \pm}}=200$ & 300 & 400 & 450 & 500 & 600 & 700 \\
\hline elel & 3.9 & 6.9 & 10 & 11 & 12 & 13 & 14 \\
\hline$\ell \ell W W$ & 7.4 & 14 & 18 & 20 & 21 & 22 & 23 \\
\hline$W W W W$ & $<0.1$ & 0.3 & 0.4 & 0.5 & 0.6 & 0.7 & 0.7 \\
\hline$\ell \ell \ell \nu$ & 40 & 55 & 66 & 68 & 70 & 72 & 74 \\
\hline$\ell \ell W Z$ & 5.2 & 9.3 & 13 & 13 & 14 & 15 & 16 \\
\hline$W W \ell \nu$ & 0.3 & 0.8 & 1.0 & 1.1 & 1.1 & 1.2 & 1.3 \\
\hline$W W W Z$ & $<0.1$ & 0.2 & 0.2 & 0.3 & 0.2 & 0.3 & 0.3 \\
\hline
\end{tabular}

Table 10. Efficiency percentages $\epsilon_{i j}^{(3 \ell)}$ for different scalar masses (in $\mathrm{GeV}$ ) and final modes $i j$ for three-lepton analyses at $\sqrt{s}=14 \mathrm{TeV}$ and the $\ell^{ \pm} \tau^{ \pm} \ell^{\mp} \nu_{\tau}\left(\tau^{\mp} \nu_{\ell}\right)$ cuts in table 6 .

energy for four and three-lepton analyses for $\mathrm{H}^{ \pm \pm} \rightarrow \ell^{ \pm} \ell^{ \pm}, W^{ \pm} W^{ \pm}$and $\mathrm{H}^{ \pm} \rightarrow \ell^{ \pm} \nu_{\ell}, W^{ \pm} Z$ for completeness, although only the efficiencies $\epsilon_{\ell \ell W W, \ell \ell W Z}^{(3 \ell)}$ for the three-lepton analysis (table 10) enter in the calculation of the LNV bounds in figure 9. 
At $7 \mathrm{TeV}$ and with an integrated luminosity $\mathcal{L}_{\text {int }}=4.9 \mathrm{fb}^{-1}$ LHC has in general no sensitivity to the LNV signals considered. But the expected bounds at $8 \mathrm{TeV}$ with $\mathcal{L}_{\text {int }}=$ $20 \mathrm{fb}^{-1}$ range from $\sim 200$ to $500 \mathrm{GeV}$ depending on the scalar multiplet. These limits can be up to $\sim 500 \mathrm{GeV}$ larger at $14 \mathrm{TeV}$ with an integrated luminosity of $100 \mathrm{fb}^{-1}$. Several comments are in order, however: $(i)$ These estimates will be improved by the experimental collaborations when cuts and efficiencies are optimized for these searches. (ii) LNV processes are in general rare and as previously emphasized, only in special regions in parameter space they are relatively large with almost half of the events from doubly-charged scalar pair and associated production violating LN. This is what we have assumed to draw figure 9. If the branching ratios are different we have to normalize the $\mathrm{H}$ cross-sections accordingly to read the corresponding limit from the figure. (iii) Relatively large LNV signals are more natural in more elaborated models, as for example, those with neutrino masses generated radiatively [29-32, 73, 74].

Two last comments are in order:

(i) LNV analyses must be improved by experimentalists not only doing a better job using real data but adapting the searches (cuts) to the signal characteristics.

(ii) On the other hand, if no departure from the SM predictions is observed, one can use all final modes to constrain the model, independently of whether LN is or is not violated or the production mechanism. The number of signal events is in this case

$$
\begin{aligned}
N^{(A)}=\mathcal{L}_{\text {int }} \times\left[\sigma_{N C} \times \sum_{i j} \mathrm{BR}\left(\mathrm{H}^{ \pm \pm} \rightarrow i\right) \times \mathrm{BR}\left(\mathrm{H}^{\mp \mp} \rightarrow j\right) \times \epsilon_{i j}^{(A)}\right. \\
\left.+\sigma_{C C} \times \sum_{r s} \mathrm{BR}\left(\mathrm{H}^{ \pm \pm} \rightarrow r\right) \times \mathrm{BR}\left(\mathrm{H}^{\mp} \rightarrow s\right) \times \epsilon_{r s}^{(A)}\right],
\end{aligned}
$$

where the sum is over all $\mathrm{H}^{ \pm \pm}(i, j, r)$ and $\mathrm{H}^{\mp}(s)$ final states contributing to analysis $A$. Here $A=4 \ell, 3 \ell$. In general, all analyses can be also taken into account together when deriving generic limits on a given model using the $\mathrm{CL}_{s}$ method by assigning each analysis to a different bin. In our case this means to 2 bins in order to account for the $4 \ell$ and $3 \ell$ analyses.

\section{Conclusions}

If neutrino masses are Majorana, LN must be violated at some scale. ${ }^{25}$ The important question at the LHC era is if it is broken at the TeV scale and hence, if LNV can be observed at the LHC. Among the simplest SM extensions which can give neutrinos a mass and predict new resonances at the LHC reach, the see-saw of type II provides the cleanest signal: in this case the mediator is a heavy scalar triplet of hypercharge $1,\left(\Delta^{++}, \Delta^{+}, \Delta^{0}\right)$, which can resonate in the same-sign dilepton channel, $\Delta^{++} \rightarrow l^{ \pm} l^{ \pm}$. The production rate is of EW size, which is the largest possible because a priori this NP has no color, and

\footnotetext{
${ }^{25}$ Independently of whether it is gauged $[115,116]$ or not.
} 
the decay products can be isolated electrons and muons with large momenta and missing energy. As these scalars have non-zero LN (equal to 2) and LN can be only very tiny broken in the SM sector, they must be pair produced. Thus, whatever the decays are, the final state must have at least four fermions typically carrying each of them one quarter of the total available energy.

In this paper we have extended the see-saw of type II and classified the scalar multiplets $\mathrm{H}$ which produce the same signals, paying special attention to their LNV decays, $p p \rightarrow \mathrm{H}^{ \pm \pm} \mathrm{H}^{\mp \mp} \rightarrow \ell^{ \pm} \ell^{ \pm} W^{\mp} W^{\mp}$ and $p p \rightarrow \mathrm{H}^{ \pm \pm} \mathrm{H}^{\mp} \rightarrow \ell^{ \pm} \ell^{ \pm} W^{\mp} Z$, not explicitly considered up to now. All those multiplets include doubly-charged scalars, being then possible to characterize $\mathrm{H}$ by the production and decay of their doubly-charged component. In particular, we have discussed the main doubly-charged scalar production mechanisms and worked out the corresponding Feynman rules in detail, providing a MadGraph5 model for Monte Carlo simulations. Finally, as a practical application we have reproduced the current searches for doubly-charged scalars by CMS and ATLAS at $\sqrt{s}=7 \mathrm{TeV}$ with $\mathcal{L}_{\text {int }}=4.9 \mathrm{fb}^{-1}[60,61]$. Present limits on their mass can be as large as $400 \mathrm{GeV}$ for the scalar triplet mediating the see-saw of type II, depending on the decay mode. These bounds raise up to $500 \mathrm{GeV}$ for the scalar multiplet of highest isospin which we have worked out, a quintuplet of hypercharge $0,\left(\Omega^{++}, \Omega^{+}, \Omega^{0}, \Omega^{-}, \Omega^{--}\right)$. Using similar cuts we have also estimated the expected bounds at $8 \mathrm{TeV}$ if no event excess is observed with an integrated luminosity of $20 \mathrm{fb}^{-1}$, being typically $150 \mathrm{GeV}$ larger than those obtained at $7 \mathrm{TeV}$. These bounds can be translated to any general model if the efficiencies for the relevant (all) channels are known. We have provided a table of Monte Carlo estimates for these efficiencies, which we have then used to estimate the bounds on LNV for different LHC runs. These limits can be near the TeV for the most favorable case at $14 \mathrm{TeV}$ with an integrated luminosity of $100 \mathrm{fb}^{-1}$ (see figure 9). However, they are only significant if the doubly-charged scalars have similar decay rates into same-sign lepton and boson pairs, as it is more natural in less simple models [29$32,73,74]$. At any rate, it must be emphasized that the analyses we have performed to obtain these bounds are not optimized for LNV searches. As a matter of fact, we have used the only analyses sensitive to doubly-charged scalar production experimentally available up to now. Hence, more sophisticated analyses taking into account the specific topology of LNV processes would have to be performed in order to extract all the information from future runs. A first attempt in this direction was given in [82-84].

We have assumed the most optimistic scenario in order to estimate the LHC potential for LNV searches. We not only assume that doubly-charged scalars have similar decay rates into same-sign lepton and gauge boson pairs, but that cascade decays within the multiplet are negligible (see footnotes 8 and 9 for further comments). Which requires that the scalar mixing is rather small [75-77]. It is thus worth to work out specific models where the optimal scenario adopted in our phenomenological approach is naturally realized.

\section{Acknowledgments}

We thank A. Santamaria and J. Wudka for previous collaboration and a careful reading of the manuscript, and J. Alcaraz and J. Santiago for useful comments. This work has been 
supported in part by the Ministry of Economy and Competitiveness (MINECO), grant FPA2010-17915, and by the Junta de Andalucía, grants FQM 101 and FQM 6552. M.C. is supported by the MINECO under the FPU program.

\section{A Contact interactions}

There can be also contact interactions generated by (even) heavier particles after integrating them out. The corresponding operators, however, are at least of dimension 6 and hence suppressed by two powers of the heavier effective scale $\Lambda$. What stands for a suppression of the doubly-charged scalar production cross-section at LHC of the order of $\left(m_{\mathrm{H}^{++}} / \Lambda\right)^{4}$.

Indeed, the contact interactions of lowest dimension must involve the gauge invariant contraction of two gluon field strength tensors $G_{a}^{\mu \nu} G_{\mu \nu}^{a}$ or a colorless quark bilinear $\bar{Q} Q^{\prime}$. In the former case the EW singlet of lowest dimension involving at least one scalar multiplet $\mathrm{H}$ with a doubly-charged component $\mathrm{H}^{++}$is $\mathrm{H}^{\dagger} \mathrm{H}$. Thus, the corresponding lowest order operator $G_{a}^{\mu \nu} G_{\mu \nu}^{a} \mathrm{H}^{\dagger} \mathrm{H}$ is of dimension 6 , being also the only one of this dimension and form.

On the other hand, there are two possible types of quark bilinears depending on the fermion chirality: $\overline{Q_{L(R)}} \gamma_{\mu} Q_{L(R)}^{\prime}$ and $\overline{Q_{L(R)}} Q_{R(L)}^{\prime}$, where $Q_{L}^{(\prime)}=q_{L}$ is the left-handed quark doublet and $Q_{R}^{(\prime)}=u_{R}, d_{R}$ are the corresponding right-handed singlets. But there is no invariant product of any of them with only one scalar multiplet $\mathrm{H}$ coupling the quark bilinear to the $\mathrm{H}$ doubly-charged component in the unitary gauge, and hence contributing to doubly-charged scalar production at hadron colliders. Besides, the vector quark bilinear requires an additional covariant derivative to ensure that the operator is Lorentz invariant. Then, in this case there is only one invariant operator of lowest dimension involving two $\mathrm{H}$ multiplets and not suppressed by a small quark Yukawa coupling: $\mathrm{H}^{\dagger}\left(D^{\mu} \mathrm{H}\right) \overline{Q_{L(R)}} \gamma_{\mu} Q_{L(R)}^{\prime}$. Other operators of dimension 6 with the covariant derivative acting on the quark fields can be shown to be suppressed by a small quark Yukawa coupling using the equations of motion; while the operator with the covariant derivative acting on the other scalar multiplet can be written as a combination of all the other operators integrating by parts.

The lowest order operators involving the other quark bilinear and two $\mathrm{H}$ multiplets are also of dimension 6 because they must involve at least a $\phi$ factor to render the operator invariant under isospin transformations. Thus, all such operators resemble $\bar{q}_{L} \phi u_{R} \mathrm{H}^{\dagger} \mathrm{H}$, being hence suppressed after EWSB by a $v / m_{\mathrm{H}^{++}}$factor relative to $\mathrm{H}^{\dagger}\left(D^{\mu} \mathrm{H}\right) \overline{Q_{L(R)}} \gamma_{\mu} Q_{L(R)}^{\prime}$. In summary, the largest contribution (at least formally) results from the operators of this form which can be, for instance, obtained after integrating out a heavy $Z^{\prime}$. But their contribution is in general suppressed far away from the heavy resonance by a $\left(m_{\mathrm{H}^{++}} / \Lambda\right)^{2}$ factor (as it is the case for $G_{a}^{\mu \nu} G_{\mu \nu}^{a} \mathrm{H}^{\dagger} \mathrm{H}$, too).

\section{B Simulation and analyses}

As indicated in the text, the signal is simulated using MadGraph5 [104] supplemented with the corresponding UFO model including the scalar interactions (the scalar gauge couplings $\mathcal{S S V}$ and $\mathcal{S S V V}$ in eq. (3.3), and the scalar couplings to fermions $\mathcal{S F \mathcal { F }}$ and to gauge bosons $\mathcal{S} \mathcal{V} \mathcal{V}$ in eqs. (2.5) and (3.4), respectively). The UFO model can be found in 
http://cafpe.ugr.es/index.php/pages/other/software in the package LNV-Scalars_UFO.tar.gz. The doubly (singly) charged scalar components for the singlet, doublet, triplet, quadruplet and quintuplet are identified by hs2, hd2, ht2, hq2 and hk2 ( ${ }^{26}$ hd1, ht1, hq1 and hk1), respectively. In addition, the package contains a set of Param Cards for the seven masses considered in our analysis, as well as a README file with examples for the production of doubly-charged scalars belonging to different multiplets and for several processes.

\section{C $\quad \mathrm{CL}_{s}$ method}

We use the $\mathrm{CL}_{s}$ method for the calculation of the exclusion limits [117]. This method associates to a sample with $\mathrm{N}$ bins the statistic

$$
\begin{aligned}
Q & =\prod_{i} \frac{\left(s_{i}+b_{i}\right)^{\tilde{n}_{i}} e^{-\left(s_{i}+b_{i}\right)}}{b_{i}^{\tilde{n}_{i}} e^{-b_{i}}} \\
& =e^{-\sum_{i} s_{i}} \prod_{i}\left[1+\frac{s_{i}}{b_{i}}\right]^{\tilde{n}_{i}},
\end{aligned}
$$

where $b_{i}$ and $s_{i}$ are the number of predicted background events and of expected signal events for bin $i$, respectively, and $\tilde{n}_{i}$ is the Poisson-distributed variable with mean $s_{i}+b_{i}$ $\left(b_{i}\right)$ for the signal+background (background-only) hypothesis. The confidence estimators

$$
\begin{aligned}
\mathrm{CL}_{s+b} & =1-\int_{Q_{\mathrm{obs}}}^{\infty} P_{s+b}(Q) d Q \quad \text { and } \\
\mathrm{CL}_{b} & =1-\int_{Q_{\mathrm{obs}}}^{\infty} P_{b}(Q) d Q
\end{aligned}
$$

are then defined integrating the corresponding density functions $P_{s+b}(Q)$ and $P_{b}(Q)$, respectively, up to $Q_{\text {obs }}$, which is the $Q$ value for $\tilde{n}_{i}$ equal to the number of observed events $n_{i}$. Thus, parameter space regions excluded at the $95 \%$ confidence level (C.L.) can be obtained requiring that $\mathrm{CL}_{s} \equiv \mathrm{CL}_{s+b} / \mathrm{CL}_{b} \leq 0.05$.

Either $Q$ or $\log Q$ can be used as statistic, although the latter is more convenient for calculating $\mathrm{CL}_{s}$ if there is only one bin (counting experiment). In this case,

$$
Q=e^{-s}\left(1+\frac{s}{b}\right)^{\tilde{n}} \Rightarrow \log Q=-s+\tilde{n}\left(1+\frac{s}{b}\right) .
$$

Hence, $\log Q$ is distributed as $\tilde{n}$ up to a scale factor and a shift. But none of them changes the ratio of areas defining $\mathrm{CL}_{s}$, being then easier to use the $\tilde{n}$ distribution as statistic.

Open Access. This article is distributed under the terms of the Creative Commons Attribution License (CC-BY 4.0), which permits any use, distribution and reproduction in any medium, provided the original author(s) and source are credited.

\footnotetext{
${ }^{26}$ Since the singlet has no singly-charged component, no name is assigned for this case.
} 


\section{References}

[1] ATLAS collaboration, Observation of a new particle in the search for the Standard Model Higgs boson with the ATLAS detector at the LHC, Phys. Lett. B 716 (2012) 1 [arXiv:1207.7214] [INSPIRE].

[2] ATLAS collaboration, Evidence for the spin-0 nature of the Higgs boson using ATLAS data, Phys. Lett. B 726 (2013) 120 [arXiv:1307.1432] [INSPIRE].

[3] CMS collaboration, Observation of a new boson at a mass of $125 \mathrm{GeV}$ with the CMS experiment at the LHC, Phys. Lett. B 716 (2012) 30 [arXiv:1207.7235] [INSPIRE].

[4] CMS collaboration, Study of the Mass and Spin-Parity of the Higgs Boson Candidate Via Its Decays to Z Boson Pairs, Phys. Rev. Lett. 110 (2013) 081803 [arXiv:1212.6639] [INSPIRE].

[5] P.P. Giardino, K. Kannike, I. Masina, M. Raidal and A. Strumia, The universal Higgs fit, arXiv: 1303.3570 [INSPIRE].

[6] J. Ellis and T. You, Updated Global Analysis of Higgs Couplings, JHEP 06 (2013) 103 [arXiv:1303.3879] [INSPIRE].

[7] A. Djouadi and G. Moreau, The couplings of the Higgs boson and its CP properties from fits of the signal strengths and their ratios at the 7+8 TeV LHC, arXiv:1303.6591 [INSPIRE].

[8] G. Bélanger, B. Dumont, U. Ellwanger, J.F. Gunion and S. Kraml, Global fit to Higgs signal strengths and couplings and implications for extended Higgs sectors, Phys. Rev. D 88 (2013) 075008 [arXiv: 1306.2941] [INSPIRE].

[9] LHC Higgs Cross Section Working Group collaboration, S. Heinemeyer et al., Handbook of LHC Higgs Cross Sections: 3. Higgs Properties, arXiv:1307.1347 [INSPIRE].

[10] R. Barbieri and A. Strumia, The 'LEP paradox', hep-ph/0007265 [INSPIRE].

[11] F. del Aguila and J. de Blas, Electroweak constraints on new physics, Fortsch. Phys. 59 (2011) 1036 [arXiv:1105.6103] [INSPIRE].

[12] Gfitter Group collaboration, M. Goebel, Status of the global fit to electroweak precisions data, PoS(ICHEP 2010) 570 [arXiv:1012.1331] [INSPIRE].

[13] ALEPH, CDF, D0, DELPHI, L3, OPAL AND SLD collaborations, LEP and TEVATRON Electroweak Working Group, SLD Electroweak and Heavy Flavour Groups, Precision Electroweak Measurements and Constraints on the Standard Model, arXiv:1012.2367 [INSPIRE].

[14] M. Baak, M. Goebel, J. Haller, A. Hoecker, D. Kennedy et al., The Electroweak Fit of the Standard Model after the Discovery of a New Boson at the LHC, Eur. Phys. J. C 72 (2012) 2205 [arXiv: 1209.2716] [INSPIRE].

[15] A. Falkowski, F. Riva and A. Urbano, Higgs at last, JHEP 11 (2013) 111 [arXiv:1303.1812] [INSPIRE].

[16] J. de Blas, Electroweak limits on physics beyond the Standard Model, EPJ Web Conf. 60 (2013) 19008 [arXiv:1307.6173] [INSPIRE].

[17] Particle Data Group collaboration, J. Beringer et al., Review of Particle Physics (RPP), Phys. Rev. D 86 (2012) 010001 [InSPIRE]. 
[18] R.N. Mohapatra and P.B. Pal, Massive neutrinos in physics and astrophysics, second edition, World Sci. Lect. Notes Phys. 60 (1998) 1 [third edition, World Sci. Lect. Notes Phys. 72 (2004) 1].

[19] S. Weinberg, Baryon and Lepton Nonconserving Processes, Phys. Rev. Lett. 43 (1979) 1566 [INSPIRE].

[20] W.H. Furry, On transition probabilities in double beta-disintegration, Phys. Rev. 56 (1939) 1184 [INSPIRE].

[21] J.D. Vergados, The Neutrinoless double beta decay from a modern perspective, Phys. Rept. 361 (2002) 1 [hep-ph/0209347] [INSPIRE].

[22] F.T. Avignone III, S.R. Elliott and J. Engel, Double Beta Decay, Majorana Neutrinos and Neutrino Mass, Rev. Mod. Phys. 80 (2008) 481 [arXiv:0708.1033] [InSPIRE].

[23] A.S. Barabash, Double beta decay experiments, Phys. Part. Nucl. 42 (2011) 613 [arXiv:1107.5663] [INSPIRE].

[24] J. Schechter and J.W.F. Valle, Neutrinoless Double beta Decay in $\mathrm{SU}(2) \times \mathrm{U}(1)$ Theories, Phys. Rev. D 25 (1982) 2951 [INSPIRE].

[25] K.S. Babu and C.N. Leung, Classification of effective neutrino mass operators, Nucl. Phys. B 619 (2001) 667 [hep-ph/0106054] [INSPIRE].

[26] K.-w. Choi, K.S. Jeong and W.Y. Song, Operator analysis of neutrinoless double beta decay, Phys. Rev. D 66 (2002) 093007 [hep-ph/0207180] [INSPIRE].

[27] J. Engel and P. Vogel, Effective operators for double beta decay, Phys. Rev. C 69 (2004) 034304 [nucl-th/0311072] [INSPIRE].

[28] A. de Gouvêa and J. Jenkins, A Survey of Lepton Number Violation Via Effective Operators, Phys. Rev. D 77 (2008) 013008 [arXiv:0708.1344] [INSPIRE].

[29] F. del Aguila, A. Aparici, S. Bhattacharya, A. Santamaria and J. Wudka, A realistic model of neutrino masses with a large neutrinoless double beta decay rate, JHEP 05 (2012) 133 [arXiv:1111.6960] [INSPIRE].

[30] F. del Aguila, A. Aparici, S. Bhattacharya, A. Santamaria and J. Wudka, Effective Lagrangian approach to neutrinoless double beta decay and neutrino masses, JHEP 06 (2012) 146 [arXiv: 1204.5986] [INSPIRE].

[31] F. del Águila, A. Aparici, S. Bhattacharya, A. Santamaria and J. Wudka, Neutrinoless double $\beta$ decay with small neutrino masses, PoS(Corfu2012)028 [arXiv:1305.4900] [INSPIRE].

[32] M. Gustafsson, J.M. No and M.A. Rivera, The Cocktail Model: Neutrino Masses and Mixings with Dark Matter, Phys. Rev. Lett. 110 (2013) 211802 [arXiv:1212.4806] [INSPIRE].

[33] R. Franceschini and R.N. Mohapatra, Radiatively Induced Type II seesaw and Vector-like 5/3 Charge Quarks, arXiv:1306.6108 [INSPIRE].

[34] W.-Y. Keung and G. Senjanović, Majorana Neutrinos and the Production of the Right-handed Charged Gauge Boson, Phys. Rev. Lett. 50 (1983) 1427 [InSPIRE].

[35] M. Fukugita and T. Yanagida, Baryogenesis Without Grand Unification, Phys. Lett. B 174 (1986) 45 [INSPIRE]. 
[36] S. Davidson, E. Nardi and Y. Nir, Leptogenesis, Phys. Rept. 466 (2008) 105 [arXiv:0802.2962] [INSPIRE].

[37] G.C. Branco, R.G. Felipe and F.R. Joaquim, Leptonic CP-violation, Rev. Mod. Phys. 84 (2012) 515 [arXiv: 1111.5332] [inSPIRE].

[38] T. Hambye, Leptogenesis: beyond the minimal type-I seesaw scenario, New J. Phys. 14 (2012) 125014 [arXiv:1212.2888] [INSPIRE].

[39] P. Minkowski, $\mu \rightarrow$ er at a Rate of One Out of 1-Billion Muon Decays?, Phys. Lett. B 67 (1977) 421 [INSPIRE].

[40] T. Yanagida, Horizontal Symmetry And Masses Of Neutrinos, in proceedings of Workshop on the Unified Theories and the Baryon Number in the Universe, Tsukuba, Japan, 13-14 Feb 1979 [INSPIRE].

[41] M. Gell-Mann, P. Ramond and R. Slansky, Complex Spinors and Unified Theories, in proceedings of Supergravity Workshop, 27-28 Sep. 1979, Stony Brook, New York, USA [arXiv:1306.4669] [INSPIRE].

[42] S. L. Glashow, The Future Of Elementary Particle Physics, NATO Adv. Study Inst. Ser. B Phys. 59 (1980) 687 [INSPIRE].

[43] R.N. Mohapatra and G. Senjanović, Neutrino Mass and Spontaneous Parity Violation, Phys. Rev. Lett. 44 (1980) 912 [INSPIRE].

[44] J. Schechter and J.W.F. Valle, Neutrino Masses in SU(2) x U(1) Theories, Phys. Rev. D 22 (1980) 2227 [INSPIRE].

[45] M. Magg and C. Wetterich, Neutrino Mass Problem and Gauge Hierarchy, Phys. Lett. B 94 (1980) 61 [INSPIRE].

[46] T.P. Cheng and L.-F. Li, Neutrino Masses, Mixings and Oscillations in $\mathrm{SU}(2) \times \mathrm{U}(1)$ Models of Electroweak Interactions, Phys. Rev. D 22 (1980) 2860 [INSPIRE].

[47] G.B. Gelmini and M. Roncadelli, Left-Handed Neutrino Mass Scale and Spontaneously Broken Lepton Number, Phys. Lett. B 99 (1981) 411 [inSPIRE].

[48] G. Lazarides, Q. Shafi and C. Wetterich, Proton Lifetime and Fermion Masses in an SO(10) Model, Nucl. Phys. B 181 (1981) 287 [InSPIRE].

[49] R.N. Mohapatra and G. Senjanović, Neutrino Masses and Mixings in Gauge Models with Spontaneous Parity Violation, Phys. Rev. D 23 (1981) 165 [inSPIRE].

[50] R. Foot, H. Lew, X.G. He and G.C. Joshi, Seesaw Neutrino Masses Induced by a Triplet of Leptons, Z. Phys. C 44 (1989) 441 [InSPIRE].

[51] E. Ma, Pathways to naturally small neutrino masses, Phys. Rev. Lett. 81 (1998) 1171 [hep-ph/9805219] [INSPIRE].

[52] A. Hektor, M. Kadastik, M. Muntel, M. Raidal and L. Rebane, Testing neutrino masses in little Higgs models via discovery of doubly charged Higgs at LHC, Nucl. Phys. B 787 (2007) 198 [arXiv: 0705.1495] [INSPIRE].

[53] F. del Aguila and J.A. Aguilar-Saavedra, Distinguishing seesaw models at LHC with multi-lepton signals, Nucl. Phys. B 813 (2009) 22 [arXiv:0808.2468] [INSPIRE].

[54] T. Han and B. Zhang, Signatures for Majorana neutrinos at hadron colliders, Phys. Rev. Lett. 97 (2006) 171804 [hep-ph/0604064] [INSPIRE]. 
[55] F. del Aguila, J.A. Aguilar-Saavedra and R. Pittau, Heavy neutrino signals at large hadron colliders, JHEP 10 (2007) 047 [hep-ph/0703261] [INSPIRE].

[56] A. Atre, T. Han, S. Pascoli and B. Zhang, The Search for Heavy Majorana Neutrinos, JHEP 05 (2009) 030 [arXiv:0901.3589] [InSPIRE].

[57] R. Franceschini, T. Hambye and A. Strumia, Type-III see-saw at LHC, Phys. Rev. D 78 (2008) 033002 [arXiv:0805.1613] [INSPIRE].

[58] F. del Aguila and J.A. Aguilar-Saavedra, Electroweak scale seesaw and heavy Dirac neutrino signals at LHC, Phys. Lett. B 672 (2009) 158 [arXiv:0809.2096] [InSPIRE].

[59] A. Arhrib et al., Collider Signatures for Heavy Lepton Triplet in Type I+III Seesaw, Phys. Rev. D 82 (2010) 053004 [arXiv: 0904.2390] [INSPIRE].

[60] CMS collaboration, A search for a doubly-charged Higgs boson in pp collisions at $\sqrt{s}=7$ TeV, Eur. Phys. J. C 72 (2012) 2189 [arXiv:1207.2666] [INSPIRE].

[61] ATLAS collaboration, Search for doubly-charged Higgs bosons in like-sign dilepton final states at $\sqrt{s}=7 \mathrm{TeV}$ with the ATLAS detector, Eur. Phys. J. C 72 (2012) 2244 [arXiv:1210.5070] [INSPIRE].

[62] F. del Aguila, J. de Blas and M. Pérez-Victoria, Effects of new leptons in Electroweak Precision Data, Phys. Rev. D 78 (2008) 013010 [arXiv:0803.4008] [INSPIRE].

[63] P.S.B. Dev, A. Pilaftsis and U.-k. Yang, New Production Mechanism for Heavy Neutrinos at the $L H C$, arXiv: 1308.2209 [INSPIRE].

[64] CMS collaboration, Search for heavy Majorana neutrinos in $\mu^{+} \mu^{+}\left[\mu^{-} \mu^{-}\right]$and $e^{+} e^{+}\left[e^{-} e^{-}\right]$ events in pp collisions at $\sqrt{s}=7$ TeV, Phys. Lett. B 717 (2012) 109 [arXiv:1207.6079] [INSPIRE].

[65] ATLAS collaboration, Search for Majorana neutrino production in pp collisions at $\sqrt{s}=7$ $\mathrm{TeV}$ in dimuon final states with the ATLAS detector, ATLAS-CONF-2012-139 (2012).

[66] J.C. Pati and A. Salam, Lepton Number as the Fourth Color, Phys. Rev. D 10 (1974) 275 [Erratum ibid. D 11 (1975) 703-703] [INSPIRE].

[67] R.N. Mohapatra and J.C. Pati, A Natural Left-Right Symmetry, Phys. Rev. D 11 (1975) 2558 [INSPIRE].

[68] G. Senjanović and R.N. Mohapatra, Exact Left-Right Symmetry and Spontaneous Violation of Parity, Phys. Rev. D 12 (1975) 1502 [INSPIRE].

[69] S.N. Gninenko, M.M. Kirsanov, N.V. Krasnikov and V.A. Matveev, Detection of heavy Majorana neutrinos and right-handed bosons, Phys. Atom. Nucl. 70 (2007) 441 [INSPIRE].

[70] F. del Aguila, J.A. Aguilar-Saavedra and J. de Blas, Trilepton signals: the golden channel for seesaw searches at LHC, Acta Phys. Polon. B 40 (2009) 2901 [arXiv:0910.2720] [INSPIRE].

[71] P.S.B. Dev, C.-H. Lee and R.N. Mohapatra, Natural TeV-Scale Left-Right Seesaw for Neutrinos and Experimental Tests, Phys. Rev. D 88 (2013) 093010 [arXiv:1309.0774] [INSPIRE].

[72] CMS collaboration, Search for heavy neutrinos and W[R] bosons with right-handed couplings in a left-right symmetric model in pp collisions at $\sqrt{s}=7 \mathrm{TeV}$, Phys. Rev. Lett. 109 (2012) 261802 [arXiv:1210.2402] [INSPIRE]. 
[73] K.S. Babu, S. Nandi and Z. Tavartkiladze, New Mechanism for Neutrino Mass Generation and Triply Charged Higgs Bosons at the LHC, Phys. Rev. D 80 (2009) 071702 [arXiv:0905.2710] [INSPIRE].

[74] G. Bambhaniya, J. Chakrabortty, S. Goswami and P. Konar, Generation of Neutrino mass from new physics at TeV scale and Multi-lepton Signatures at the LHC, Phys. Rev. D 88 (2013) 075006 [arXiv: 1305.2795] [INSPIRE].

[75] J.A. Grifols, A. Mendez and G.A. Schuler, Production and Decay of Doubly Charged Higgs Bosons of Left-Right Symmetric Models, Mod. Phys. Lett. A 4 (1989) 1485 [InSPIRE].

[76] A. Djouadi, J. Kalinowski and P.M. Zerwas, Two and three-body decay modes of SUSY Higgs particles, Z. Phys. C 70 (1996) 435 [hep-ph/9511342] [INSPIRE].

[77] P. Fileviez Perez, T. Han, G.-y. Huang, T. Li and K. Wang, Neutrino Masses and the CERN LHC: Testing Type II Seesaw, Phys. Rev. D 78 (2008) 015018 [arXiv:0805.3536] [INSPIRE].

[78] M. Aoki, S. Kanemura and K. Yagyu, Testing the Higgs triplet model with the mass difference at the LHC, Phys. Rev. D 85 (2012) 055007 [arXiv:1110.4625] [INSPIRE].

[79] J. Hisano and K. Tsumura, Higgs boson mixes with an $\mathrm{SU}(2)$ septet representation, Phys. Rev. D 87 (2013) 053004 [arXiv: 1301.6455] [InSPIRE].

[80] M. Raidal, A. van der Schaaf, I. Bigi, M.L. Mangano, Y.K. Semertzidis et al., Flavour physics of leptons and dipole moments, Eur. Phys. J. C 57 (2008) 13 [arXiv:0801.1826] [INSPIRE].

[81] P. Nath, B.D. Nelson, H. Davoudiasl, B. Dutta, D. Feldman et al., The Hunt for New Physics at the Large Hadron Collider, Nucl. Phys. Proc. Suppl. 200-202 (2010) 185 [arXiv: 1001.2693 ] [INSPIRE].

[82] F. del Aguila, M. Chala, A. Santamaria and J. Wudka, Discriminating between lepton number violating scalars using events with four and three charged leptons at the LHC, Phys. Lett. B 725 (2013) 310 [arXiv:1305.3904] [INSPIRE].

[83] F. del Aguila, M. Chala, A. Santamaria and J. Wudka, Distinguishing between lepton number violating scalars at the LHC, EPJ Web Conf. 60 (2013) 17002 [arXiv:1307.0510] [INSPIRE].

[84] F. del Aguila, M. Chala, A. Santamaria and J. Wudka, Lepton Number Violation and Scalar Searches at the LHC, Acta Phys. Polon. B 44 (2013) 2139 [arXiv:1311.2950] [InSPIRE].

[85] A. Alloul, M. Frank, B. Fuks and M.R. de Traubenberg, Doubly-charged particles at the Large Hadron Collider, Phys. Rev. D 88 (2013) 075004 [arXiv: 1307.1711] [INSPIRE].

[86] K.S. Babu, Model of 'Calculable' Majorana Neutrino Masses, Phys. Lett. B 203 (1988) 132 [INSPIRE].

[87] J.F. Gunion, C. Loomis and K.T. Pitts, Searching for doubly charged Higgs bosons at future colliders, eConf C 960625 (1996) LTH096 [hep-ph/9610237] [INSPIRE].

[88] V. Rentala, W. Shepherd and S. Su, A Simplified Model Approach to Same-sign Dilepton Resonances, Phys. Rev. D 84 (2011) 035004 [arXiv:1105.1379] [INSPIRE].

[89] M. Aoki, S. Kanemura and K. Yagyu, Doubly-charged scalar bosons from the doublet, Phys. Lett. B 702 (2011) 355 [Erratum ibid. B 706 (2012) 495] [arXiv:1105.2075] [INSPIRE].

[90] K. Yagyu, Higgs sectors with exotic scalar fields, arXiv:1304.6338 [INSPIRE]. 
[91] B. Ren, K. Tsumura and X.-G. He, A Higgs Quadruplet for Type III Seesaw and Implications for $\mu \rightarrow e \gamma$ and $\mu-e$ Conversion, Phys. Rev. D 84 (2011) 073004 [arXiv:1107.5879] [INSPIRE].

[92] C.-W. Chiang, T. Nomura and K. Tsumura, Search for doubly charged Higgs bosons using the same-sign diboson mode at the LHC, Phys. Rev. D 85 (2012) 095023 [arXiv: 1202.2014] [INSPIRE].

[93] A.G. Akeroyd and M. Aoki, Single and pair production of doubly charged Higgs bosons at hadron colliders, Phys. Rev. D 72 (2005) 035011 [hep-ph/0506176] [INSPIRE].

[94] T. Han, B. Mukhopadhyaya, Z. Si and K. Wang, Pair production of doubly-charged scalars: Neutrino mass constraints and signals at the LHC, Phys. Rev. D 76 (2007) 075013 [arXiv: 0706.0441] [INSPIRE].

[95] E.J. Williams, Nature of the high-energy particles of penetrating radiation and status of ionization and radiation formulae, Phys. Rev. 45 (1934) 729 [INSPIRE].

[96] E.J. Williams, Nature of the high-energy particles of penetrating radiation and status of ionization and radiation formulae, Phys. Rev. 45 (1934) 729 [INSPIRE].

[97] M. Drees, R.M. Godbole, M. Nowakowski and S.D. Rindani, $\gamma \gamma$ processes at high-energy pp colliders, Phys. Rev. D 50 (1994) 2335 [hep-ph/9403368] [INSPIRE].

[98] B. Gripaios, A. Pomarol, F. Riva and J. Serra, Beyond the Minimal Composite Higgs Model, JHEP 04 (2009) 070 [arXiv:0902 .1483] [INSPIRE].

[99] J. Mrazek, A. Pomarol, R. Rattazzi, M. Redi, J. Serra and A. Wulzer, The Other Natural Two Higgs Doublet Model, Nucl. Phys. B 853 (2011) 1 [arXiv:1105.5403] [InSPIRE].

[100] E. Bertuzzo, T.S. Ray, H. de Sandes and C.A. Savoy, On Composite Two Higgs Doublet Models, JHEP 05 (2013) 153 [arXiv:1206.2623] [INSPIRE].

[101] M. Chala, $h \rightarrow \gamma \gamma$ excess and Dark Matter from Composite Higgs Models, JHEP 01 (2013) 122 [arXiv: 1210.6208] [INSPIRE].

[102] L. Vecchi, The Natural Composite Higgs, arXiv:1304.4579 [INSPIRE].

[103] M. Muhlleitner and M. Spira, A Note on doubly charged Higgs pair production at hadron colliders, Phys. Rev. D 68 (2003) 117701 [hep-ph/0305288] [InSPIRE].

[104] J. Alwall, M. Herquet, F. Maltoni, O. Mattelaer and T. Stelzer, MadGraph 5: Going Beyond, JHEP 06 (2011) 128 [arXiv:1106.0522] [INSPIRE].

[105] N.D. Christensen and C. Duhr, FeynRules - Feynman rules made easy, Comput. Phys. Commun. 180 (2009) 1614 [arXiv:0806.4194] [INSPIRE].

[106] H. Sugiyama, K. Tsumura and H. Yokoya, Discrimination of models including doubly charged scalar bosons by using tau lepton decay distributions, Phys. Lett. B 717 (2012) 229 [arXiv:1207.0179] [inSPIRE].

[107] S. Kanemura, K. Yagyu and H. Yokoya, First constraint on the mass of doubly-charged Higgs bosons in the same-sign diboson decay scenario at the LHC, Phys. Lett. B 726 (2013) 316 [arXiv: 1305.2383] [INSPIRE].

[108] C. Englert, E. Re and M. Spannowsky, Pinning down Higgs triplets at the LHC, Phys. Rev. D 88 (2013) 035024 [arXiv: 1306.6228] [INSPIRE].

[109] T. Sjöstrand, S. Mrenna and P.Z. Skands, PYTHIA 6.4 Physics and Manual, JHEP 05 (2006) 026 [hep-ph/0603175] [INSPIRE]. 
[110] S. Ovyn, X. Rouby and V. Lemaitre, DELPHES, a framework for fast simulation of a generic collider experiment, arXiv:0903.2225 [INSPIRE].

[111] M. Cacciari, G.P. Salam and G. Soyez, FastJet User Manual, Eur. Phys. J. C 72 (2012) 1896 [arXiv:1111.6097] [INSPIRE].

[112] E. Conte, B. Fuks and G. Serret, MadAnalysis 5, A User-Friendly Framework for Collider Phenomenology, Comput. Phys. Commun. 184 (2013) 222 [arXiv:1206.1599] [INSPIRE].

[113] R. Frederix, S. Frixione, V. Hirschi, F. Maltoni, R. Pittau and P. Torrielli, Four-lepton production at hadron colliders: aMC@NLO predictions with theoretical uncertainties, JHEP 02 (2012) 099 [arXiv:1110.4738] [INSPIRE].

[114] S. Frixione and B.R. Webber, Matching NLO QCD computations and parton shower simulations, JHEP 06 (2002) 029 [hep-ph/0204244] [INSPIRE].

[115] M. Duerr, P. Fileviez Perez and M.B. Wise, Gauge Theory for Baryon and Lepton Numbers with Leptoquarks, Phys. Rev. Lett. 110 (2013) 231801 [arXiv:1304.0576] [INSPIRE].

[116] P. Fileviez Perez and M.B. Wise, Low Scale Quark-Lepton Unification, Phys. Rev. D 88 (2013) 057703 [arXiv: 1307.6213] [INSPIRE].

[117] A.L. Read, Presentation of search results: The CL(s) technique, J. Phys. G 28 (2002) 2693 [INSPIRE]. 